\title{
Non-Homogeneous Semi-Markov and Markov Renewal Processes and Change of Measure in Credit Risk
}

\author{
P.-C.G. Vassiliou
}

check for updates

Citation: Vassiliou, P.-C.G. NonHomogeneous Semi-Markov and Markov Renewal Processes and Change of Measure in Credit Risk. Mathematics 2021, 9, 55.

https://doi.org/10.3390/math901 0055

Received: 18 November 2020 Accepted: 22 December 2020 Published: 29 December 2020

Publisher's Note: MDPI stays neutral with regard to jurisdictional clai$\mathrm{ms}$ in published maps and institutional affiliations.

Copyright: (C) 2020 by the author. Licensee MDPI, Basel, Switzerland. This article is an open access article distributed under the terms and conditions of the Creative Commons Attribution (CC BY) license (https:// creativecommons.org/licenses/by/ $4.0 /)$.
Department of Statistical Sciences, University College London, Gower Street, London WC1E 6BT, UK; vasiliou@math.auth.gr

\begin{abstract}
For a $\mathcal{G}$-inhomogeneous semi-Markov chain and $\mathcal{G}$-inhomogeneous Markov renewal processes, we study the change from real probability measure into a forward probability measure. We find the values of risky bonds using the forward probabilities that the bond will not default up to maturity time for both processes. It is established in the form of a theorem that the forward probability measure does not alter the semi Markov structure. In addition, foundation of a $\mathcal{G}_{-}$ inhohomogeneous Markov renewal process is done and a theorem is provided where it is proved that the Markov renewal process is maintained under the forward probability measure. We show that for an inhomogeneous semi-Markov there are martingales that characterize it. We show that the same is true for a Markov renewal processes. We discuss in depth the calibration of the $\mathcal{G}$-inhomogeneous semi-Markov chain model and propose an algorithm for it. We conclude with an application for risky bonds.
\end{abstract}

Keywords: credit rating; non-homogeneous semi-Markov chains; non-homogeneous Markov renewal processes; change of measure

MSC: Primary 05C38; 15A15

\section{Introduction}

The migration process is the evolution of the credit quality of corporate bonds, corporate liabilities, corporate loans, etc. Credit rating models in credit risk management have flourished considerably in recent years.

These models known also as migration models represent the evolution of the rating of a company or a state. They do so in order to evaluate the default risk of a bond or a loan, or the term structure of both and constitutes an important issue for risk management and pricing. The commercial rating agencies such as Moody's Investor Service and Standard and Poor's et al. determine the credit classes or sometimes it is done internally. The modeling of the migration process is an important issue for risk management and pricing. Rating transition matrices are of particular interest for determining the economic capital figures like expected loss and VAR for credit portfolios, but also can be helpful as it comes to the pricing of more complex products in the credit industry.

The modeling of the evolution of credit migration started with a simple Markov chain model either in discrete or continuous time. Carty and Fons [1], with data spanning from 1976 to 1993 from Moody's Investor service, showed that the Weibull distribution was the appropriate one to represent the time spent in a credit class. Thus, in fact, they established using real data that the Markov chain model was not realistic. A generation of models followed dominated by duration analysis, and an important study in this area which could act also as a link to these class of models is Duffie et al. [2].

More recently, inhomogeneous semi-Markov processes have been proposed for the migration process as more realistic models for the variability of the rating transition matrices by Vasileiou and Vassiliou [3]. D' Amico et al. [4] proposed a homogeneous Markov 
renewal model for the evolution of the credit migration process. The difference of the semi-Markov approach as defined in Howard [5] and the Markov renewal approach is basically on the basic parameters chosen to build the model. D' Amico et al. [6,7] introduced non-homogeneous semi-Markov of reliability theory type models; in $\mathrm{D}^{\prime}$ Amico [8], a semi-Markov maintenance model with imperfect repair at random times was introduced; in D' Amico et al. $[9,10]$, the backward and forward non-homogeneous semi-Markov process was introduced and studied to some extent.; in Vassiliou and Vasileiou [11], an inhomogeneous semi-Markov process is introduced to study the asymptotic behavior of the survival probabilities; in Vassiliou [12], fuzzy sets are introduced to face the problem that rating agencies disagree with the majority of their ratings. The new stochastic and mathematical problems created with the introduction of fuzzy sets are being answered mainly for the quasi-stationarity problem. The above publications of non-homogeneous semi-Markov and Markov renewal processes for credit risk was followed by quite a lot of literature by the same and other authors in credit risk and related subjects. For example, from recent years, see Huang [13], D' Amico et al. [14,15], D' Amico [16], Magni et al. [17], Wu et al. [18,19], Puneet et al. [20], De Blasis [21]. In D' Amico et al. [22], bivariate semi-Markov processes are introduced for the pricing of Credit Default Swaps (CDS). The Credit Default Swap (CDS) is a bilateral agreement that transfers credit risk between two contractual parties, protection buyer (that faces credit risk from a third party), and protection seller. Finally, Vassiliou [23] introduced the idea of the stochastic Market environment to express the changes in the general economy, which affect any industry in small or great amounts of turbulence.

The vast majority of the existing models for the migration process use a discrete time setting. The roots of this tendency is the fact that the most prominent risk management tools, such as J.P. Morgan's Credit Metrics and McKinsey's Credit Portfolio View, are built around estimates of rating migration probabilities. In essence, the estimates by these agencies and in the published academic literature use a discrete-time setting and rely on a "cohort" method that estimates the transition rates. In what follows, our semi-Markov chain and the related Markov renewal chain will be in a discrete-time setting. Lando and Skodeberg [24] argued that the estimation of the transition probabilities in their proposed continuous time setting among other advantages is getting a better grip on the rare events. However, the results in the present are independent from the method used to estimate the transition probabilities that is the discrete time method used in Vasileiou et al. [3] and the continuous time method proposed by Lando and Skodeberg [24]. Note also that the continuous time method of estimation of the transition probabilities is easily transferred from the Markov chain model of Lando and Skodeberg [24] in the present non-homogeneous semiMarkov chain and Markov renewal models while retaining the apparent advantages of the later models. The general problem of obtaining the results that follow in a continuous time setting altogether is an interesting and challenging mathematical problem for future research probably in the near future.

In Section 2, we provide what is already known but necessary in what follows. The Market $M_{d}$ of the savings account, the default free zero-coupon bond, and the defaultable zero-coupon bond are defined. We also introduce the Randon-Nikodym derivatives under the real probability, the equivalent martingale, and the forward martingale measures. Finally, the need to study the change to forward the martingale measure is shown for the evolution of defaultable bonds in the various grades. This is done by providing the price of defaultable bonds and their spreads as functions of the forward probabilities.

Section 3 introduces a new definition of the $\mathcal{G}$-inhomogeneous semi-Markov process than the last in Vasileiou et al [3]. The differences brought about into the new definition, although they are not apparently essential, are the ones needed in order that: $(i)$ to state theorem 1, which establishes the change from the real world probabilities to forward probabilities in an inhomogeneous semi-Markov process; (ii) to introduce the necessary definitions, theorems, and all the results and algorithms that follow in the present paper. 
Section 4 introduces a new definition of the $\mathcal{G}$-inhomogeneous Markov renewal process. Markov renewal processes have been used in many studies (see D'Amico et al. [4,6-8] as a model for the migration process using the real probabilities. In theorem 2, we state and prove that the change of measure under certain conditions retains the Markov renewal property. In addition, we provide under the same conditions functional relationships between the real world transition probabilities and the forward transition probabilities.

In Section 5 and in Theorem 3, we provide a new martingale characterization, for the discrete time $\mathcal{G}$-inhomogeneous semi-Markov process. This characterization apart from its general interest will prove to be very useful in Sections 7 and 8 .

In Section 6 and in Theorem 4, we provide a new martingale characterization, for the discrete time $\mathcal{G}$-inhomogeneous Markov renewal process.

We alter in Section 7 the conditions of Theorem 1, in a way that the theorem will still be valid under the new conditions. The new conditions are more restrictive but still remain quite general. However, they will be useful and in Theorem 5 we establish new interesting simplified closed analytic relations among the forward probability measure transition probability sequences and the corresponding real world probability measure ones under the new conditions.

In Section 8, we study the calibration of the $\mathcal{G}$-inhomogeneous semi-Markov model. In Section 8.1, we make the new risk premium assumptions and provide their consequences on the results of Theorem 5. In Section 8.2, we introduce the real and forward entrance probability measures and establish stochastic difference equations among the real entrance probability measures. In addition, functional relationships are established between the forward entrance probability measures and the default free zero-coupon bonds and the defaultable zero-coupon bonds. In Section 8.3, we propose Algorithm 8.1 for the evaluation of the needed forward entrance probability measures. The algorithm, although similar in nature and perception as the algorithm in Section 6 of Vasileiou and Vassiliou [3], also serves the purpose of clarifying many details. In addition, by understanding in some depth the present Algorithm 8.1, we could go back to the algorithm in Section 6 of Vasileiou and Vassiliou [3] and easily make some needed corrections.

In Section 9, we provide an illustrative application of the inhomogeneous semi-Markov model in the evolution of the migration process in credit risk. The forward entrance probabilities are evaluated in a classical problem using representative data.

\section{A General Discrete-Time Market Model}

Let a complete filtered probability space $\left(\Omega, \mathbb{G}, \mathbb{Q}, \mathcal{G}_{t}\right)$ with $\mathbb{Q}$ being the real-world probability measure which represents the actual probability of events in $\Omega, \mathbb{G}$ the $\sigma$-algebra, and $\mathcal{G}_{t}$ a filtration in $\mathbb{G}$ where the variable $t$ is discrete and represents the time, and is being generated by the economic assets to be modeled in the filtered probability space. Let the time set $\mathbb{T}=\{0,1,2, \ldots, T\}$, where $T$ is the terminal date of the economic assets, that is, the trading horizon. The points of $\mathbb{T}$ are the admissible trading dates.

The Market $M_{d}$. Let $\left\{r_{t}\right\}_{t=0}^{\infty}$ be the discrete time stochastic processes which is the interest rate of the savings account in the market, and it is assumed to be adapted to the filtration $\mathcal{G}_{t}$. The savings account of the market $M_{d}$ has a value at time $t$ given by

$$
B_{t}=\prod_{u=0}^{t-1}\left(1+r_{u}\right), \text { with } B_{0}=1
$$

In addition, in the market $M_{d}$, the default free zero-coupon bond whose price is $B(t, T)$ for $t=0,1, \ldots, T$ is included. Let that $k$ rating grades are distinguished by the rating agencies for a defaultable bond. Define by $D_{i}(t, T)$ the price process of a defaultable bond of grate $i$ for specific $T \in \mathbb{T}$ and $t=0,1, \ldots, T, i=1,2, \ldots, k$. We call the market with the above economic assets the market $M_{d}$, and we assume that it is perfect, i.e., all assets in market $M_{d}$ are perfectly divisible and the market is frictionless. 
Let the column vector $\mathbf{D}(t, T)$ represent the price process of the assets in market $M_{d}$ :

$$
\mathbf{D}(t, T)=\left[B_{t}^{(0)}, B^{(1)}(t, T), D_{1}^{(2)}(t, T), D_{2}^{(3)}(t, T), \ldots, D_{k}^{(k+1)}(t, T)\right]^{\top} .
$$

By using as numéraire the savings account, we get the column vector

$$
\tilde{\mathbf{D}}(t, T)=\left[1, \beta_{t} B^{(1)}(t, T), \beta_{t} D_{1}^{(2)}(t, T), \beta_{t} D_{2}^{(3)}(t, T), \ldots, \beta_{t} D_{k}^{(k+1)}(t, T)\right],
$$

with $\beta_{t}=1 / B_{t}^{(0)}$, and is called the discounted price process of the market.

Let $\mathbb{Q}^{*}$ be an equivalent martingale measure equivalent with the real probability measure $\mathbb{Q}$. It is known then that $\mathbb{Q}^{*}$ is such that the discounted bond price $\tilde{B}(t, T)=$ $B(t, T) / B_{t}$, for $0 \leq t \leq T$ and all possible values of $T$ are a martingale under $\mathbb{Q}^{*}$ and in relation with the filtration $\mathcal{G}_{t}$. Analogously, a $\mathbb{Q}^{*}$ is an equivalent martingale measure for the market $M_{d}$, if it is an equivalent martingale measure for the discounted asset price process $\tilde{\mathbf{D}}(t, T)$. How to construct such equivalent martingale measures is known and could be found, for example, in Bingham and Kiesel [25], Shreve [26] and Vassiliou [27].

We assume that the market $M_{d}$ is viable and complete. Then, it is known (Vassiliou et al. [23]) that the equivalent martingale measure $\mathbb{Q}^{*}$ is unique for the market $M_{d}$ and guarantees the existence of the forward martingale measure $\mathbb{Q}_{T}$. We have seen that $\mathbb{Q}^{*}$ is an equivalent martingale measure for the discounted bond price $\tilde{B}(t, T)$, hence it is known that

$$
B(t, T)=B_{t} \mathbb{E}_{\mathbb{Q}^{*}}\left(B_{T}^{-1} \mid \mathcal{G}_{t}\right), t \in[0, T]
$$

Now, let the Radon-Nikodym derivative

$$
\left.\frac{d \mathbb{Q}^{*}}{d \mathbb{Q}}\right|_{\mathcal{G}_{T}}=\psi_{T} \quad \mathbb{Q} \text {-a.s., }
$$

where the $\mathcal{G}_{T}$-measurable random variable $\psi_{T}$ is strictly positive $\mathbb{Q}$-a.s. and $\mathbb{E}_{\mathbb{Q}}\left(\psi_{T}\right)=1$. Then, the density process $\psi_{t}=\mathbb{E}_{\mathbb{Q}}\left(\psi_{T} \mid \mathcal{G}_{t}\right), t=0,1, \ldots, T$, follows a strictly positive martingale under $\mathbb{Q}$. It is known that the forward measure $\mathbb{Q}_{T}$ on $\left(\Omega, \mathcal{G}_{T}\right)$ with $T$ the trading horizon or equivalently the forward martingale measure $\mathbb{Q}_{T}$ on $\left(\Omega, \mathcal{G}_{T}\right)$ is equivalent to $\mathbb{Q}^{*}$, and their Radon-Nikodym derivative is equal to

$$
\frac{d \mathbb{Q}_{T}}{d \mathbb{Q}^{*}}=\frac{B_{T}^{-1}}{\mathbb{E}_{\mathbb{Q}^{*}}\left(B_{T}^{-1}\right)}=\frac{1}{B_{T} B(0, T)} \mathbb{Q}^{*}-\text { a.s. }
$$

When restricted to the $\sigma$-algebra $\mathcal{G}_{t}$, we get that

$$
\eta_{t}:=\left.\frac{d \mathbb{Q}_{T}}{d \mathbb{Q}^{*}}\right|_{\mathcal{G}_{t}}=\mathbb{E}_{\mathbb{Q}^{*}}\left(\left.\frac{1}{B_{T} B(0, T)}\right|_{\mathcal{G}_{t}}\right)=\frac{B(t, T)}{B_{t} B(0, T)}
$$

for every $t \in[0, T]$.

From (6) and (7), we find that

$$
\left.\frac{d \mathbb{Q}_{T}}{d \mathbb{Q}}\right|_{\mathcal{G}_{T}}=\frac{\psi_{T}}{B_{T} B(0, T)}=\theta_{T} \quad \mathbb{Q} \text {-a.s. }
$$

where the $\mathcal{G}_{T}$-measurable random variable $\theta_{T}$ is strictly positive $\mathbb{Q}$-a.s. and $\mathbb{E}_{\mathbb{Q}}\left(\theta_{T}\right)=1$. It is known that the density process $\theta_{t}=\mathbb{E}_{\mathbb{Q}}\left(\theta_{T} \mid \mathcal{G}_{t}\right), t=0,1, \ldots, T$, follows a strictly positive martingale under $\mathbb{Q}$.

Let $D^{\delta}(t, T)$ be the process of price at time $t$ of a defaultable bond. Then, from Bielecki and Rutkowski [28] or Vasileiou and Vassiliou [3], we obtain

$$
D^{\delta}(t, T)=B_{t} \mathbb{E}_{\mathbb{Q}^{*}}\left(B_{T}^{-1}\left(\delta \mathbf{1}_{\{T \geq \tau\}}+\mathbf{1}_{\{T<\tau\}}\right) \mid \mathcal{G}_{t}\right),
$$


where $\mathbf{1}_{\{T \geq \tau\}}$, which is equal to 1 if $T \geq \tau$ and equals 0 in all other cases, $\tau$ is the default time and $\delta$ is the recovery rate. Following Vasileiou and Vassiliou [3] p. 176, we have that

$$
\begin{gathered}
D^{\delta}(t, T)=B(t, T) \mathbb{E}_{\mathbb{Q}_{T}}\left(\left(\delta \mathbf{1}_{\{T \geq \tau\}}+\mathbf{1}_{\{T<\tau\}}\right) \mid \mathcal{G}_{t}\right) \\
=B(t, T)\left(\delta+(1-\delta) \mathbb{Q}_{T}\left\{\tau>T \mid \mathcal{G}_{t}\right\}\right) .
\end{gathered}
$$

It is apparent that it is important to estimate the probability of default under the forward probability measure. Among other reasons, it allows us to relax the assumption of constant interest rate and have instead an interest rate process $r_{t}$. By definition, the forward rate of one time step of the risky bond at time $T$, as seen from $t \leq T$, is given by

$$
f_{D}(t, T)=-\log \left(\frac{D^{\delta}(t, T+1)}{D^{\delta}(t, T)}\right) .
$$

Analogously, for the default-free bond, we have

$$
f(t, T)=-\log \left(\frac{B(t, T+1)}{B(t, T)}\right)
$$

It follows that the credit spread process will be given by

$$
s(t, T)=f_{D}(t, T)-f(t, T)=\log \left(\frac{\delta+(1-\delta) \mathbb{Q}_{T}\left\{\tau>T \mid \mathcal{G}_{t}\right\}}{\delta+(1-\delta) \mathbb{Q}_{T+1}\left\{\tau>T+1 \mid \mathcal{G}_{t}\right\}}\right) .
$$

Apparently, the above is true for any credit state i. We then have

$$
D_{i}^{\delta}(t, T)=B(t, T) \mathbb{E}_{\mathbb{Q}_{T}}\left(\left(\delta \mathbf{1}_{\{T \geq \tau\}}+\mathbf{1}_{\{T<\tau\}}\right) \mid X_{t}=i\right), i=1,2, \ldots, k .
$$

From the above relations, it is apparent that it is necessary to calculate the probability $\mathbb{Q}_{T}\left\{\tau>T \mid X_{t}\right\}$. Therefore, we need to study the change to forward the martingale measure for the model we will use for the evolution of defaultable bonds in the various states.

\section{The $\mathcal{G}$ Inhomogeneous Semi-Markov Process and Change to Forward Martingale Measure}

In [3], a different and novel definition of an inhomogeneous semi-Markov process appeared than the one in [29]. The delicate essential differences in the cores of the two definitions were those that made possible the Proof of Theorem 4.1, p.179 in Vasileiou and Vassiliou [3], which dealt with the change of measure from the real world probability measure to the forward probability measure in an inhomogeneous semi-Markov process. In the present section, we will amend the definition of an inhomogeneous semi-Markov process. The differences brought into the new definition, although they do not seem essential, are the ones that will allow the existence and use of Theorem 1 in the present, and the definitions, theorems, and all the results and algorithms that follow in the present paper.

Let $\left\{X_{t}\right\}_{t=0}^{\infty}$ be a stochastic process on the complete filtered probability space $\left(\Omega, \mathbb{G}, \mathbb{Q}, \mathcal{G}_{t}\right)$, with state space $\mathbb{K}=\{1,2, \ldots, k, k+1\}$, where $k$ is the the number of grades of a defaultable bond, and $k+1$ the default state. The value $X_{t}=i$ means that the defaultable bond enters state $i$ at time $t$. Denote by $\mathbb{F}^{X}$ the natural filtration of $\left\{X_{t}\right\}_{t=0}^{\infty}$ and assume $\mathbb{F}^{X} \subseteq \mathbb{G}$. Now, let $S_{t+1}$ that represents the choice of movement in the interval $[t, t+1)$ of the defaultable bond, given that it last entered its present grade at time $t$. Let the natural filtration of the process $\left\{S_{t}\right\}_{t=0}^{\infty}$ be denoted by $\mathbb{F}^{S}$ and assume that $\mathbb{F}^{S} \mathrm{VF} \mathbb{F}^{X} \subseteq \mathbb{G}$. The pair $\left\{X_{t}, S_{t+1}\right\}_{t=0}^{\infty}$ is a discrete-time $\mathbb{G}$-inhomogeneous semi-Markov process if what follows holds: 
(a) The process $\left\{S_{t+1}\right\}_{t=0}^{\infty}$ is a non-homogeneous Markov chain adapted to $\left\{X_{t}\right\}_{t=0}^{\infty}$ with respect to $\mathbb{G}$ under $\mathbb{Q}$, that is, if $f: \mathbb{K} \rightarrow \mathbb{R}$ is any function, then

$$
\mathbb{E}_{\mathbb{Q}}\left(f\left(S_{t+n}\right) \mid \mathcal{G}_{t}\right)=\mathbb{E}_{\mathbb{Q}}\left(f\left(S_{t+n}\right) \mid \sigma\left(X_{t}\right)\right), \quad t, n=0,1,2, \ldots
$$

Denote by $p_{i j}(t)=\mathbb{Q}\left\{S_{t+1}=j \mid X_{t}=i\right\}$ and $\widetilde{\mathbf{P}}(t)=\left\{p_{i j}(t)\right\}_{i, j \in \mathbb{K}}$ the transition probabilities with

$$
\widetilde{\mathbf{P}}(t)=\left(\begin{array}{ccccc}
p_{11}(t) & p_{12}(t) & \ldots & p_{1 k}(t) & p_{1, k+1}(t) \\
p_{21}(t) & p_{22}(t) & \ldots & p_{2 k}(t) & p_{2, k+1}(t) \\
\cdots & \ldots & \ldots & \ldots & \ldots \\
p_{k 1}(t) & p_{k 2}(t) & \cdots & p_{k k}(t) & p_{k, k+1}(t) \\
0 & 0 & \ldots & 0 & 1
\end{array}\right)=\left(\begin{array}{cc}
\mathbf{P}(t) & \mathbf{p}_{k+1}^{\top}(t) \\
0 & 1
\end{array}\right)
$$

(b) According to (a), whenever a defaultable bond enters state $\mathrm{i}$ at time $\mathrm{t}$, it chooses credit class j, and moves to it with $p_{i j}(t)=\mathbb{Q}\left\{S_{t+1}=j \mid X_{t}=i\right\}$. Now, before performing the actual transition from credit class i to credit class $\mathrm{j}$, it "holds" for a time $\omega_{i j}(t)$ in state $i$. All $\omega_{i j}(t)$ are positive, integer-valued random variables with a probability mass function $h_{i j}(t, m)$, where

$$
h_{i j}(t, m)=\mathbb{Q}\left\{\omega_{i j}(t)=m \mid S_{t+1}=j, X_{t}=i\right\}, \quad t, m=0,1,2, \ldots
$$

The matrix $\widetilde{\mathbf{H}}(t, m)=\left\{h_{i j}(t, m)\right\}_{i, j \in \mathbb{K}}$ is of the form

$$
\begin{gathered}
\widetilde{\mathbf{H}}(t, m)=\left(\begin{array}{ccccc}
h_{11}(t, m) & h_{12}(t, m) & \ldots & h_{1 k}(t, m) & h_{1, k+1}(t, m) \\
h_{21}(t, m) & h_{22}(t, m) & \ldots & h_{2 k}(t, m) & h_{2, k+1}(t, m) \\
\ldots & \ldots & \ldots & \ldots & \ldots \\
h_{k 1}(t, m) & h_{k 2}(t, m) & \ldots & h_{k k}(t, m) & h_{k, k+1}(t, m) \\
0 & 0 & \ldots & \ldots & \mathbf{1}_{\{m=1\}}
\end{array}\right)= \\
=\left(\begin{array}{ccc}
\mathbf{H}(t, m) & \mathbf{h}_{k+1}^{\top}(t, m) \\
\mathbf{0} & \mathbf{1}_{\{m=1\}}
\end{array}\right) .
\end{gathered}
$$

This means that all elements of $\widetilde{\mathbf{H}}(t, m)$ are assumed finite and $\widetilde{\mathbf{H}}(t, 0)=0$.

(c) Let $f: \mathbb{K} \times \mathbb{K} \times \mathbb{N}^{*} \rightarrow \mathbb{R}$ be any function, then

$$
\begin{gathered}
\mathbb{E}\left\{f\left(X_{t}, S_{t+1}, \omega_{X_{t}, S_{t+1}}(t)\right) \mid \mathcal{G}_{t+1}\right\}= \\
\mathbb{E}\left\{f\left(X_{t}, S_{t+1}, \omega_{X_{t}, S_{t+1}}(t)\right) \mid \sigma\left(X_{t}\right) V \sigma\left(S_{t+1}\right)\right\},
\end{gathered}
$$

where $\mathbb{N}^{*}=\{0,1,2, \ldots\}$.

We call $\left\{S_{t+1}\right\}_{t=0}^{\infty}$ the inherent $\mathbb{G}$-inhomogeneous Markov process.

Our next goal is to examine the changes brought into Theorem 4.1 p. 179 in Vasileiou and Vassiliou [3] by the changes, made in the present, in the definition of an inhomogeneous semi-Markov processes. Following the steps of the Proof of Theorem 4.1 in Vasileiou and Vassiliou [3], it is possible to show that, as expected, the preservation of the $\mathbb{G}$-inhomogeneous semi-Markov property remains, the relationships between the realworld probabilities $p_{i j}(t)=\mathbb{Q}\left\{S_{t+1}=j \mid X_{t}=i\right\}$ and probability mass functions

$$
h_{i j}(t, m)=\mathbb{Q}\left\{\omega_{i j}(t)=m \mid S_{t+1}=j, X_{t}=i\right\},
$$

to the respective probabilities under $\mathbb{Q}_{T}$, are essentially the same, but the conditions under which both results hold changes accordingly. These results are stated in the following theorem, the proof of which is omitted due to the similarity with Theorem $4.1 \mathrm{p} .179$ in Vasileiou and Vassiliou [3]: 
Theorem 1. We assume the random variable $\theta_{t}^{-1} \theta_{t+1}$ be $\left(\sigma\left(X_{t}\right) V \sigma\left(S_{t+1}\right)\right)$-measurable for any $t=0,1, \ldots, T-1$.Consequently, for all values of $t$ :

$$
\theta_{t}^{-1} \theta_{t+1}=g_{t}\left(X_{t}, S_{t+1}\right)
$$

for any function $g_{t}: \mathbb{K} \times \mathbb{K} \rightarrow \mathbb{R}$. In addition, we assume that $\theta_{t}^{-1} \theta_{t+\omega_{X_{t}, S_{t+1}}(t)}$ is $\left(\sigma\left(X_{t}\right) V \sigma\left(S_{t+1}\right) V \sigma\left(\omega_{X_{t}, S_{t+1}}(t)\right)\right)$-measurable for any $t=0,1, \ldots, T-1$. Equivalently,

$$
\theta_{t+1}^{-1} \theta_{t+\omega_{X}, S_{t+1}(t)}=f_{t+1}\left(X_{t}, S_{t+1}, \omega_{X_{t}, S_{t+1}}(t)\right)
$$

for some function $f_{t+1}: \mathbb{K} \times \mathbb{K} \times \mathbb{N}^{*} \rightarrow \mathbb{R}$. Assuming that $\left\{X_{t}, S_{t+1}\right\}_{t=0}^{\infty}$ follows a discretetime $\mathbb{G}$-inhomogeneous semi-Markov process under $\mathbb{Q}$, then a discrete-time $\mathbb{G}$-inhomogeneous semi-Markov process under $\mathbb{Q}_{T}$ also follows, and, in addition,

$$
\begin{gathered}
(a)_{F} p_{i j}(t):=\mathbb{Q}_{T}\left\{S_{t+1}=j \mid X_{t}=i\right\}=g_{t}(i, j) p_{i j}(t) \\
(b){ }_{F} h_{i j}(t, m)=\mathbb{Q}_{T}\left\{\omega_{i j}(t)=m \mid S_{t+1}=j, X_{t}=i\right\}=f_{t+1}(i, j, m) h_{i j}(t, m) .
\end{gathered}
$$

\section{The $\mathcal{G}$ Non-Homogeneous Markov Renewal Process and Change to Forward Martingale Measure}

The above definition of the $\mathcal{G}$-non-homogeneous semi-Markov process was based on the definition of the non-homogeneous semi-Markov process given in Vassiliou and Papadopoulou (1992), which had its roots on the definition of the homogeneous semiMarkov process given in Howard [5]. Pyke [30] provided the definition and preliminary properties of the homogeneous Markov renewal process. The two stochastic processes are equivalent; however, the basic parameters defining the processes are quite different. Many authors have used homogeneous and inhomogeneous Markov renewal processes for the immigration process of defaultable bonds (see D'Amico et al. [4,6-8]). Therefore, there is a need to define an $\mathcal{G}$-inhomogeneous Markov renewal process and study in detail the change to forward martingale measure of their founding parameters.

Let $\left(\Omega, \mathbb{G}, \mathbb{Q}, \mathcal{G}_{t}\right)$ be a complete filtered probability space, and $\left\{Y_{n}\right\}_{n=0}^{\infty}$ a stochastic process taking values in the state space $\mathbb{K}=\{1,2, \ldots, k, k+1\}$ and, representing the credit state, the defaultable bond enters at the $n$-th jump. Let $\mathbb{F}^{Y}$ the natural filtration of $\left\{Y_{n}\right\}_{n=0}^{\infty}$ and assume that it is a subfiltration of $\mathbb{G}$. Let $\left\{T_{n}\right\}$ be the family of random variables taking values in $\mathbb{R}^{+}=[0, \infty)$ such that $0 \leq T_{0} \leq T_{1} \leq \ldots$ expressing the time of the $n$-th jump $\left(T_{n}\right)$. Let $\mathbb{F}^{T}$ be the natural filtration of $\left\{T_{n}\right\}_{n=0}^{\infty}$ and allow that $\mathbb{F}^{T} \vee \mathbb{F}^{Y} \subseteq \mathbb{G}$.

The stochastic process $\left\{Y_{n}, T_{n}\right\}$ is said to be a non-homogeneous Markov renewal process provided that, for every bounded or non-negative function $f: \mathbb{K} \rightarrow \mathbb{R}$, we have

$$
\begin{gathered}
\mathbb{E}_{\mathbb{Q}}\left(f\left(Y_{n}, Y_{n+1}, T_{n+1}-T_{n}, T_{n}\right) \mid \mathcal{G}_{n}\right)= \\
\mathbb{E}_{\mathbb{Q}}\left(f\left(Y_{n}, Y_{n+1}, T_{n+1}-T_{n}, T_{n}\right) \mid \sigma\left(Y_{n}\right) \vee \sigma\left(T_{n}\right)\right),
\end{gathered}
$$

for $n=0,1,2, \ldots$. Define by

$$
\mathbb{Q}\left\{Y_{n+1}=j, T_{n+1}-T_{n} \leq x \mid Y_{n}=i, T_{n}=t\right\}=Q(i, j, x, t) .
$$

The family of probabilities $Q(i, j, x, t)$ for $i, j \in \mathbb{K}, x \in \mathbb{R}^{+}, T \in \mathbb{N}$ is called a nonhomogeneous Markov kernel.

The function $x \rightarrow Q(i, j, x, t)$ has all the properties of a distribution function except that

$$
p_{i j}(t)=\lim _{x \rightarrow \infty} Q(i, j, x, t)=Q(i, j, \infty, t) .
$$


Indeed, one could easily find that

$$
p_{i j}(t) \geq 0, \sum_{j \in \mathbb{K}} p_{i j}(t)=1 .
$$

If $p_{i j}(t)=0$ for some pair $(i, j)$, then $Q(i, j, x, t)=0$ for all $x$; we then define $Q(i, j, x, t) / p_{i j}(t)=1$. With this convention, we define

$$
\mathbb{G}(i, j, x, t)=\frac{Q(i, j, x, t)}{p_{i j}(t)} \text { for } i, j \in \mathbb{K}, x \in \mathbb{R}^{+}, T \in \mathbb{N} .
$$

From (17) and (20), we get that

$$
\mathbb{G}(i, j, x, t)=\mathbb{Q}\left\{T_{n+1}-T_{n} \leq x \mid Y_{n}=i, Y_{n+1}=j, T_{n}=t\right\} .
$$

Remark 1. Note that, in words, the increments $T_{1}-T_{0}$ and $T_{2}-T_{1}$ in the inhomogeneous case, unlike the homogeneous, are not conditionally independent, given the Markov chain $Y_{0}, Y_{1}, Y_{2}$ and the $T_{0}, T_{1}$ jumping times.

We will need the following Lemma from Musiela and Rutkowski [31].

Lemma 1. (Abstract version of Bayes Formula). Let $\mathcal{G}$ be a sub $\sigma$-field of the $\sigma$-field $\mathbb{G}$. Now, consider $\psi$ a random variable integrable in relation to $\mathbb{Q}$; then, $\mathbb{Q}_{T}$ is a probability measure equivalent to $\mathbb{Q}$ with

$$
\frac{d \mathbb{Q}_{T}}{d \mathbb{Q}} \mid \mathcal{G}_{T}=\eta_{T}, \mathbb{Q} \text {-a.s., }
$$

with the random variable $\eta_{T}$ strictly positive $\mathbb{Q}$-a.s.; moreover, $\eta_{T}$ is $\mathbb{Q}$ integrable with $\mathbb{E}_{\mathbb{Q}}\left(\eta_{T}\right)=$ 1. Then,

$$
\mathbb{E}_{\mathbb{Q}_{T}}\left(\psi \mid \mathcal{G}_{t}\right)=\frac{\mathbb{E}_{\mathbb{Q}}\left(\psi \eta \mid \mathcal{G}_{t}\right)}{\mathbb{E}_{\mathbb{Q}}\left(\psi \mid \mathcal{G}_{t}\right)}
$$

With the use of the above Lemma, we prove the following basic theorem:

Theorem 2. Let $\left(\Omega, \mathbb{G}, \mathbb{Q}, \mathcal{G}_{t}\right)$ be a complete filtered probability space and $\left\{Y_{n}, T_{n}\right\}$ a nonhomogeneous Markov renewal process with Markov kernel $Q(i, j, x, t)$ given by (4.2). In addition, let the random variable $\theta_{T_{n}}^{-1} \theta_{T_{n+1}}$ be $\sigma\left(Y_{n}\right) \vee \sigma\left(Y_{n+1}\right) \vee \sigma\left(T_{n}\right) \vee \sigma\left(T_{n+1}-T_{n}\right)$ measurable for all $T_{n}=0,1, \ldots, T-1$. Then, for every $T_{n}$,

$$
\theta_{T_{n}}^{-1} \theta_{T_{n+1}}=F_{T_{n}}\left(Y_{n}, Y_{n+1}, T_{n+1}-T_{n}\right) .
$$

The pair $\left\{Y_{n}, T_{n}\right\}_{n=0}^{\infty}$ follows a $\mathbb{G}$-non-homogeneous Markov renewal process under $\mathbb{Q}_{T}$ and moreover

$$
{ }_{F} Q(i, j, x, t)=F_{t}(i, j, x) Q(i, j, x, t),
$$

where ${ }_{F} Q(i, j, x, t)$ is the transition probability matrix of the forward probabilities.

Proof. Using Lemma 1, we fix $T_{n}$ and, for any $j \in \mathbb{K}$, we get

$$
\begin{gathered}
\mathbb{Q}^{\top}\left\{Y_{n+1}=j, T_{n+1}-T_{n} \leq x \mid \mathcal{G}_{T_{n}}\right\}=\mathbb{E}_{\mathbb{Q}^{\top}}\left\{\mathbf{1}_{\left\{Y_{n+1}=j, T_{n+1}-T_{n} \leq x\right\}} \mid \mathcal{G}_{T_{n}}\right\} \\
=\frac{\mathbb{E}_{\mathbb{Q}}\left\{\theta_{T} \mathbf{1}_{\left\{Y_{n+1}=j, T_{n+1}-T_{n} \leq x\right\}} \mid \mathcal{G}_{T_{n}}\right\}}{\mathbb{E}_{\mathbb{Q}}\left\{\theta_{T} \mid \mathcal{G}_{T_{n}}\right\}} \\
=\left(\theta_{T} \text { is a martingale under } \mathbb{Q} \text { and } \mathcal{G}_{T_{n}} \text {-measurable }\right) \\
=\mathbb{E}_{\mathbb{Q}}\left\{\theta_{T_{n}}^{-1} \theta_{T} \mathbf{1}_{\left\{Y_{n+1}=j, T_{n+1}-T_{n} \leq x\right\}} \mid \mathcal{G}_{T_{n}}\right\}
\end{gathered}
$$




$$
\begin{gathered}
=\text { (Tower property) } \\
=\mathbb{E}_{\mathbb{Q}}\left[\mathbb{E}_{\mathbb{Q}}\left\{\theta_{T_{n}}^{-1} \theta_{T} \mathbf{1}_{\left\{Y_{n+1}=j, T_{n+1}-T_{n} \leq x\right\}} \mid \mathcal{G}_{T_{n+1}}\right\} \mathcal{G}_{T_{n}}\right] \\
=\mathbb{E}_{\mathbb{Q}}\left[\theta_{T_{n}}^{-1} \mathbb{E}_{\mathbb{Q}}\left\{\theta_{T} \mid \mathcal{G}_{T_{n+1}}\right\} \mathbf{1}_{\left\{Y_{n+1}=j, T_{n+1}-T_{n} \leq x\right\}} \mid \mathcal{G}_{T_{n}}\right] \\
=\mathbb{E}_{\mathbb{Q}}\left[\theta_{T_{n}}^{-1} \theta_{T_{n+1}} \mathbf{1}_{\left\{Y_{n+1}=j, T_{n+1}-T_{n} \leq x\right\}} \mid \mathcal{G}_{T_{n}}\right] \\
=\mathbb{E}_{\mathbb{Q}}\left[F_{T_{n}}\left(Y_{n}, Y_{n+1}, T_{n+1}-T_{n}\right) \mathbf{1}_{\left\{Y_{n+1}=j, T_{n+1}-T_{n} \leq x\right\}} \mid \mathcal{G}_{T_{n}}\right] \\
=\mathbb{E}_{\mathbb{Q}}\left[f\left(Y_{n}, Y_{n+1}, T_{n+1}-T_{n}, T_{n}\right) \mid \mathcal{G}_{T_{n}}\right] \\
=(\text { by 4.1) } \\
=\mathbb{E}_{\mathbb{Q}}\left[f\left(Y_{n}, Y_{n+1}, T_{n+1}-T_{n}, T_{n}\right) \mid \sigma\left(Y_{n}\right) \vee \sigma\left(T_{n}\right)\right] .
\end{gathered}
$$

Now, (22) actually shows that $\mathbb{Q}^{\top}\left\{Y_{n+1}=j, T_{n+1}-T_{n} \leq x \mid \mathcal{G}_{T_{n}}\right\}$ is a $\sigma\left(Y_{n}\right) \vee \sigma\left(T_{n}\right)$ measurable random variable. Since $\sigma\left(Y_{n}\right) \vee \sigma\left(T_{n}\right) \subseteq \mathcal{G}_{T_{n}}$, we get that

$$
\begin{gathered}
\mathbb{Q}^{\top}\left\{Y_{n+1}=j, T_{n+1}-T_{n} \leq x \mid \mathcal{G}_{T_{n}}\right\}= \\
=\mathbb{Q}^{\top}\left\{Y_{n+1}=j, T_{n+1}-T_{n} \leq x \mid \sigma\left(Y_{n}\right) \vee \sigma\left(T_{n}\right)\right\} .
\end{gathered}
$$

Moreover, we have

$$
\begin{gathered}
{ }_{F} Q(i, j, x, t)=\mathbb{Q}^{\top}\left\{Y_{n+1}=j, T_{n+1}-T_{n} \leq x \mid Y_{n}=i, T_{n}=t\right\} \\
=\mathbb{E}_{\mathbb{Q}}\left[F_{T_{n}}\left(Y_{n}, Y_{n+1}, T_{n+1}-T_{n}\right) \mathbf{1}_{\left\{Y_{n+1}=j, T_{n+1}-T_{n} \leq x\right\}} \mid Y_{n}=i, T_{n}=t\right] \\
=F_{t}(i, j, x) Q(i, j, x, t) .
\end{gathered}
$$

\section{Martingale Characterization of the $\mathbb{G}$-Inhomogeneous Semi-Markov Process}

Martingale characterization theorems have played traditionally a basic role in the theory of stochastic process-for example, in the methodology to obtain Laws of Large numbers and Central Limit theorems. In the present section, in the form of a theorem, we will provide a martingale characterization, for the discrete time $\mathbb{G}$-inhomogeneous semi-Markov process $\left\{X_{t}, S_{t+1}\right\}_{t=0}^{\infty}$. The theorem will not be proved in the at most possible generalization, but in a form suitable for the purposes of Section 7 . In this respect, define the jump processes:

$$
H_{t}^{i}=\mathbf{1}_{\left\{X_{t}=i\right\}}, Y_{t+1}^{j}=\mathbf{1}_{\left\{S_{t+1}=j\right\}} \text { and } \Phi_{t+1}^{i j}=\mathbf{1}_{\left\{X_{t}=i, S_{t+1}=j\right\}} .
$$

and the processes

$C_{0, t}^{i j}: \quad$ the number of times the $\mathbb{G}$ - inhomogeneous semi-Markov process $\left\{X_{t}, S_{t+1}\right\}_{t=0}^{\infty}$ entered state $i$ and selected to move to state $\mathrm{j}$ in the time interval $[0, t]$.

$Z_{0, t}^{i j}(m):$ the number of transitions of the $\mathbb{G}$-inhomogeneous semi-Markov process $\left\{X_{t}, S_{t+1}\right\}_{t=0}^{\infty}$ from $i$ to $j$ that occurred in the time interval $[0, t]$ remaining time $m$ in state $i$.

Now, we arrive at the following relations for the above defined processes:

$$
C_{0, t}^{i j}=\sum_{u=0}^{t-1} H_{u}^{i} Y_{u+1}^{j} \quad, \quad Z_{0, t}^{i j}(m)=\sum_{u=0}^{t-m} \Phi_{u+1}^{i j} H_{u+m}^{j}=\sum_{u=0}^{t-m} H_{u}^{i} Y_{u+1}^{j} H_{u+m}^{j} .
$$


At this point, it is important to note that, for a discrete time $\mathbb{G}$-non-homogeneous semi-Markov process $\left\{X_{t}, S_{t+1}\right\}_{t=0}^{\infty}$, the sequences of transition probabilities $\{\widetilde{\mathbf{P}}(t)\}$ and $\{\widetilde{\mathbf{H}}(t, m)\}_{t, m=0}^{\infty}$ uniquely determine the process. In addition, together with the initial distribution, the above sequences provide sufficient information, to answer the important questions in the theory of semi-Markov processes and also the ones which proved to have great practical value in real problems.

Let $\mathbb{M}_{n, m}$ be the set of all $n \times m$ matrices with elements from $\mathbb{R}$. In addition, let $\mathbb{S M}_{n}$ be the set of $n \times n$ stochastic matrices. We now provide the following definition:

Definition 1. Let the sequence $\{\mathbf{H}(t, m)\}_{t, m}^{\infty} \in \mathbb{M}_{n, n}$; then, we say that it is an $\mathfrak{H}$-sequence if the following conditions are satisfied:

$$
\mathbf{H}(t, m) \geq 0 \text { and } \sum_{m=0}^{\infty} \mathbf{H}(t, m)=\mathbf{U}, \text { for every } t, m \in \mathbb{N}^{*}
$$

where $\mathbf{U} \in \mathbb{M}_{n, n}$ are a matrix of ones.

Theorem 3. Let there be $\left\{X_{t}, S_{t+1}\right\}_{t=0}^{\infty} \mathbb{G}$-non-homogeneous semi-Markov process, with sequences of probabilities $\{\widetilde{\mathbf{P}}(t)\}_{t=0}^{\infty}$ and $\{\widetilde{\mathbf{H}}(t, m)\}_{t, m=0}^{\infty}$. For any two states $i, j \in \mathbb{K}$, the processes

$$
\mathfrak{C}_{0, t}^{i j}=C_{0, t}^{i j}-\sum_{u=0}^{t-1} p_{i j}(u) H_{u}^{i} \text { and } \mathfrak{Y}_{0, t}^{i j}(m)=Z_{0, t}^{i j}(m)-\sum_{u=0}^{t-m} h_{i j}(u, m) \Phi_{u+1}^{i j},
$$

for $t \in \mathbb{N}^{*}$, following $\mathbb{G}$-martingales under $\mathbb{Q}$. Conversely, let

$$
\left\{\mathbf{P}^{\prime}(t)\right\}_{t=0}^{\infty} \in \mathbb{S M}_{k+1} \text { and let }\left\{\mathbf{H}^{\prime}(t, m)\right\}_{t, m=0}^{\infty} \text { an } \mathfrak{H} \text {-sequence, }
$$

then, the processes

$$
{ }^{\prime} \mathfrak{C}_{0, t}^{i j}=C_{0, t}^{i j}-\sum_{u=0}^{t-1} p_{i j}^{\prime}(u) H_{u}^{i} \text { and }{ }^{\prime} \mathfrak{Y}_{0, t}^{i j}(m)=Z_{0, t}^{i j}(m)-\sum_{u=0}^{t-m} h_{i j}^{\prime}(u, m) \Phi_{u+1}^{i j},
$$

are $\mathbb{G}$-martingales under $\mathbb{Q}$, then the sequences of matrices

$$
\{\widetilde{\mathbf{P}}(t)\}_{t=0}^{\infty} \text { and }\{\widetilde{\mathbf{H}}(t, m)\}_{t, m=0^{\prime}}^{\infty}
$$

are equal, respectively, with the sequences of matrices

$$
\left\{\mathbf{P}^{\prime}(t)\right\}_{t=0}^{\infty} \text { and }\left\{\mathbf{H}^{\prime}(t, m)\right\}_{t, m=0}^{\infty} .
$$

In conclusion, the sequences of transition probabilities

$$
\{\widetilde{\mathbf{P}}(t)\}_{t=0}^{\infty} \text { and }\{\widetilde{\mathbf{H}}(t, m)\}_{t, m=0^{\prime}}^{\infty}
$$

are the unique sequences for which the processes

$$
\left\{\mathfrak{C}_{0, t}^{i j}\right\}_{t=0}^{\infty} \text { and }\left\{\mathfrak{Y}_{0, t}^{i j}(m)\right\}_{t, m=0}^{\infty}
$$

are $\mathbb{G}$-martingales under $\mathbb{Q}$. 
Proof. It is sufficient to show :

$$
\mathbb{E}_{\mathbb{Q}}\left(\mathfrak{C}_{0, t+1}^{i j} \mid \mathcal{G}_{t}\right)=\mathfrak{C}_{0, t}^{i j} \quad \text { and } \quad \mathbb{E}_{\mathbb{Q}}\left(\mathfrak{Y}_{0, t+1}^{i j}(m) \mid \mathcal{G}_{t}\right)=\mathfrak{Y}_{0, t}^{i j}(m)
$$

We assume that $i \neq j$ and, for $i=j$, the proof will be similar. For the first part of Equation (15), we have

$$
\begin{gathered}
\mathbb{E}_{\mathbb{Q}}\left(\mathfrak{C}_{0, t+1}^{i j}-\mathfrak{C}_{0, t}^{i j} \mid \mathcal{G}_{t}\right)= \\
=\mathbb{E}_{\mathbb{Q}}\left(\sum_{u=0}^{t} H_{u}^{i} \mathrm{Y}_{u+1}^{j}-\sum_{u=0}^{t} p_{i j}(u) H_{u}^{i}-\sum_{u=0}^{t-1} H_{u}^{i} \mathrm{Y}_{u+1}^{j}+\sum_{u=0}^{t-1} p_{i j}(u) H_{u}^{i} \mid \mathcal{G}_{t}\right) \\
=\mathbb{E}_{\mathbb{Q}}\left(H_{t}^{i} \mathrm{Y}_{t+1}^{j}-p_{i j}(t) H_{t}^{i} \mid \mathcal{G}_{t}\right) \\
=\mathbb{E}_{\mathbb{Q}}\left(H_{t}^{i}\left(\mathrm{Y}_{t+1}^{j}-p_{i j}(t)\right) \mid \sigma\left(X_{t}\right)\right)=0 .
\end{gathered}
$$

Now, from the second part of Equation (28), we have

$$
\begin{gathered}
\mathbb{E}_{\mathbb{Q}}\left(\mathfrak{Y}_{0, t+1}^{i j}(m)-\mathfrak{Y}_{0, t}^{i j}(m) \mid \mathcal{G}_{t}\right)= \\
=\mathbb{E}_{\mathbb{Q}}\left(Z_{0, t+1}^{i j}(m)-\sum_{u=0}^{t-m+1} h_{i j}(u, m) \Phi_{u+1}^{i j}-Z_{0, t}^{i j}(m)+\sum_{u=0}^{t-m} h_{i j}(u, m) \Phi_{u+1}^{i j} \mid \mathcal{G}_{t}\right) \\
=\mathbb{E}_{\mathbb{Q}}\left(\begin{array}{c}
\sum_{u=0}^{t-m+1} \Phi_{u+1}^{i j} H_{u+m}^{j}-\sum_{u=0}^{t-m+1} h_{i j}(u, m) \Phi_{u+1}^{i j}-\sum_{u=0}^{t-m} \Phi_{u+1}^{i j} H_{u+m}^{j} \\
+\sum_{u=0}^{t-m} h_{i j}(u, m) \Phi_{u+1}^{i j} \mid \mathcal{G}_{t}
\end{array}\right) \\
=\mathbb{E}_{\mathbb{Q}}\left(\Phi_{t-m+2}^{i j} H_{t+1}^{j}-h_{i j}(t-m+1, m) \Phi_{t-m+2}^{i j} \mid \mathcal{G}_{t}\right) \\
=\mathbb{E}_{\mathbb{Q}}\left(H_{t-m+1}^{i} Y_{t-m+2}^{j}\left(H_{t+1}^{j}-h_{i j}(t-m+1, m)\right) \mid \mathcal{G}_{t}\right) \\
=\mathbb{E}_{\mathbb{Q}}\left(H_{t-m+1}^{i} Y_{t-m+2}^{j}\left(H_{t+1}^{j}-h_{i j}(t-m+1, m)\right) \mid \sigma\left(X_{t}\right) V \sigma\left(S_{t+1}\right)\right)=0 .
\end{gathered}
$$

Conversely, since ${ }^{\prime} \mathfrak{C}_{0, t}^{i j}$ is a martingale under $\mathbb{Q}$, we have that

$$
\mathbb{E}_{\mathbb{Q}}\left(\mathfrak{C}_{0, t+1}^{i j}-{ }^{\prime} \mathfrak{C}_{0, t}^{i j} \mid \mathcal{G}_{t}\right)=0
$$

Consequently,

$$
\begin{gathered}
\mathbb{E}_{\mathbb{Q}}\left(\sum_{u=0}^{t} H_{u}^{i} \mathrm{Y}_{u+1}^{j}\right. \\
\left.-\sum_{u=0}^{t} p_{i j}^{\prime}(u) H_{u}^{i}-\sum_{u=0}^{t-1} H_{u}^{i} \mathrm{Y}_{u+1}^{j}+\sum_{u=0}^{t-1} p_{i j}^{\prime}(u) H_{u}^{i} \mid \mathcal{G}_{t}\right) \\
=\mathbb{E}_{\mathbb{Q}}\left(H_{t}^{i} \mathrm{Y}_{t+1}^{j}-p_{i j}^{\prime}(t) H_{t}^{i} \mid \mathcal{G}_{t}\right)=0 .
\end{gathered}
$$

From Equations (29) and (31), we get

$$
\mathbb{E}_{\mathbb{Q}}\left(H_{t}^{i} Y_{t+1}^{j}-p_{i j}(t) H_{t}^{i}-H_{t}^{i} Y_{t+1}^{j}+p_{i j}^{\prime}(t) H_{t}^{i} \mid \mathcal{G}_{t}\right)=0,
$$

or

$$
\mathbb{E}_{\mathbb{Q}}\left(p_{i j}^{\prime}(t) H_{t}^{i}-p_{i j}(t) H_{t}^{i} \mid \mathcal{G}_{t}\right)=\mathbb{E}_{\mathbb{Q}}\left(p_{i j}^{\prime}(t) H_{t}^{i}-p_{i j}(t) H_{t}^{i} \mid \sigma\left(X_{t}\right)\right)=0,
$$

from which we conclude that

$$
\mathbf{P}^{\prime}(t)=\widetilde{\mathbf{P}}(t) \text { for every } t \in \mathbb{N}^{*}
$$


Since ' $\mathfrak{Y}_{0, t}^{i j}(m)$ is a martingale under $\mathbb{Q}$, we have that

$$
\mathbb{E}_{\mathbb{Q}}\left(\mathfrak{Y}_{0, t+1}^{i j}(m)-{ }^{\prime} \mathfrak{Y}_{0, t}^{i j}(m) \mid \mathcal{G}_{t}\right)=0
$$

Consequently,

$$
\begin{gathered}
\mathbb{E}_{\mathbb{Q}}\left(Z_{0, t+1}^{i j}(m)-\sum_{u=0}^{t-m+1} h_{i j}^{\prime}(u, m) \Phi_{u+1}^{i j}-Z_{0, t}^{i j}(m)+\sum_{u=0}^{t-m} h_{i j}^{\prime}(u, m) \Phi_{u+1}^{i j} \mid \mathcal{G}_{t}\right) \\
=\mathbb{E}_{\mathbb{Q}}\left(\Phi_{t-m+2}^{i j} H_{t+1}^{j}-h_{i j}^{\prime}(t-m+1, m) \Phi_{t-m+2}^{i j} \mid \mathcal{G}_{t}\right) \\
=\mathbb{E}_{\mathbb{Q}}\left(H_{t-m+1}^{i} Y_{t-m+2}^{j}\left(H_{t+1}^{j}-h_{i j}^{\prime}(t-m+1, m)\right) \mid \mathcal{G}_{t}\right)=0
\end{gathered}
$$

From Equations (30) and (32), we get

$$
\mathbb{E}_{\mathbb{Q}}\left(\begin{array}{c}
\Phi_{t-m+2}^{i j} H_{t+1}^{j}-h_{i j}^{\prime}(t-m+1, m) \Phi_{t-m+2}^{i j}-\Phi_{t-m+2}^{i j} H_{t+1}^{j} \\
+h_{i j}(t-m+1, m) \Phi_{t-m+2}^{i j} \mid \mathcal{G}_{t}
\end{array}\right)=0
$$

or

$$
\begin{aligned}
& \mathbb{E}_{\mathbb{Q}}\left(\Phi_{t-m+2}^{i j}\left(h_{i j}(t-m+1, m)-h_{i j}^{\prime}(t-m+1, m)\right) \mid \mathcal{G}_{t}\right) \\
& =\mathbb{E}_{\mathbb{Q}}\left(H_{t-m+1}^{i} \mathrm{Y}_{t-m+2}^{j}\left(h_{i j}(t-m+1, m)-h_{i j}^{\prime}(t-m+1, m)\right) \mid \mathcal{G}_{t}\right) \\
& =\mathbb{E}_{\mathbb{Q}}\left(\begin{array}{c}
H_{t-m+1}^{i} \mathrm{Y}_{t-m+2}^{j}\left(h_{i j}(t-m+1, m)-h_{i j}^{\prime}(t-m+1, m)\right) \\
\mid \sigma\left(X_{t-1}\right) \operatorname{V\sigma }\left(S_{t}\right)
\end{array}\right)=0,
\end{aligned}
$$

from which we conclude that

$$
\widetilde{\mathbf{H}}(t, m)=\mathbf{H}^{\prime}(t, m) \text { for every } t, m \in \mathbb{N}^{*} .
$$

\section{Martingale Characterization of the $\mathbb{G}-$ Non-Homogeneous Markov Renewal Process}

We start by providing in the form of a theorem a martingale characterization, for the $\mathbb{G}$-non-homogeneous Markov renewal process $\left\{Y_{n}, T_{n}\right\}_{n=0}^{\infty}$. Define the process

$R_{0, T_{n}}^{i j}(x): \quad$ the number of transitions of the $\mathbb{G}$-IMRP from $i$ to $j$ that occurred in the interval $\left[0, T_{n}\right]$ with sojourn time to state $i$ being less than $x$.

In addition, define the jump processes

$$
\Gamma_{T_{n}}^{j}=\mathbf{1}_{\left\{Y_{T_{n}}=j\right\}} \quad, \quad D_{r}^{a}=\mathbf{1}_{\left\{T_{r+1}-T_{r}=a\right\}} .
$$

Now, we have that

$$
R_{0, T_{n}}^{i j}(x)=\sum_{a=1}^{x} \sum_{r=0}^{n-1} \Gamma_{T_{r}}^{i} \Gamma_{T_{r+1}}^{j} D_{r}^{a} .
$$

Then, we define the following stochastic process:

$$
\mathcal{R}_{0, T_{n}}^{i j}(x)=R_{0, T_{n}}^{i j}(x)-\sum_{r=0}^{n-1} Q(i, j, x, t) \Gamma_{T_{r}}^{i} \Gamma_{T_{r+1}}^{j} .
$$

We will now prove the following martingale characterization theorem for a $\mathbb{G}$ inhomogeneous Markov renewal theorem. 
Theorem 4. Let $\left(\Omega, \mathbb{G}, \mathbb{Q}, \mathcal{G}_{t}\right)$ be a complete filtered probability space and $\left\{Y_{n}, T_{n}\right\}$ a nonhomogeneous Markov renewal process with Markov kernel $Q(i, j, x, t)$ given by (17). For any two states $i, j \in \mathbb{K}$, the process

$$
\mathcal{R}_{0, T_{n}}^{i j}(x)=R_{0, T_{n}}^{i j}(x)-\sum_{r=0}^{n-1} Q\left(i, j, x, T_{r}\right) \Gamma_{T_{r}}^{i} \Gamma_{T_{r+1}}^{j}
$$

for $T_{n} \in \mathbb{N}^{*}$ follows $\mathbb{G}$-martingales under $\mathbb{Q}$. Conversely, let the inhomogeneous Markov kernel $Q^{\prime}(i, j, x, t)$; then, if

$$
\mathcal{R}_{0, T_{n}}^{\prime i j}(x)=R_{0, T_{n}}^{i j}(x)-\sum_{r=0}^{n-1} Q^{\prime}\left(i, j, x, T_{r}\right) \Gamma_{T_{r}}^{i} \Gamma_{T_{r+1}}^{j}
$$

is a $\mathbb{G}$-martingale under $\mathbb{Q}$, then the inhomogeneous Markov kernel $Q^{\prime}(i, j, x, t)$ is equal to the inhomogeneous Markov kernel $Q(i, j, x, t)$. In conclusion, the inhomogeneous Markov kernel $Q(i, j, x, t)$ is the unique Markov kernel for which the processes $\mathcal{R}_{0, T_{n}}^{i j}(x)$ are a $\mathbb{G}$-martingale under $\mathbb{Q}$.

Proof. It is sufficient to show:

$$
\mathbb{E}_{\mathbb{Q}}\left(\mathcal{R}_{0, T_{n+1}}^{i j}(x) \mid \mathcal{G}_{T_{n}}\right)=\mathcal{R}_{0, T_{n}}^{i j}(x)
$$

We assume that $i \neq j$ and for $i=j$ the proof will be similar. We get that

$$
\begin{gathered}
\mathbb{E}_{\mathbb{Q}}\left(\mathcal{R}_{0, T_{n+1}}^{i j}(x)-\mathcal{R}_{0, T_{n}}^{i j}(x) \mid \mathcal{G}_{T_{n}}\right)= \\
\mathbb{E}_{\mathbb{Q}}\left(\sum_{a=1}^{x} \sum_{r=0}^{n} \Gamma_{T_{r}}^{i} \Gamma_{T_{r+1}}^{j} D_{r}^{a}-\sum_{r=0}^{n} Q\left(i, j, x, T_{r}\right) \Gamma_{T_{r}}^{i} \Gamma_{T_{r+1}}^{j}\right. \\
\left.-\sum_{a=1}^{x} \sum_{r=0}^{n-1} \Gamma_{T_{r}}^{i} \Gamma_{T_{r+1}}^{j} D_{r}^{a}+\sum_{r=0}^{n-1} Q\left(i, j, x, T_{r}\right) \Gamma_{T_{r}}^{i} \Gamma_{T_{r+1}}^{j} \mid \mathcal{G}_{T_{n}}\right) \\
=\mathbb{E}_{\mathbb{Q}}\left(\sum_{a=1}^{x} \Gamma_{T_{n}}^{i} \Gamma_{T_{n+1}}^{j} D_{r}^{a}-Q\left(i, j, x, T_{n}\right) \Gamma_{T_{n}}^{i} \Gamma_{T_{n+1}}^{j} \mid \mathcal{G}_{T_{n}}\right) \\
=\mathbb{E}_{\mathbb{Q}}\left[\Gamma_{T_{n}}^{i} \Gamma_{T_{n+1}}^{j}\left(\sum_{a=1}^{x} D_{r}^{a}-Q\left(i, j, x, T_{n}\right)\right) \mid \mathcal{G}_{T_{n}}\right] \\
=\mathbb{E}_{\mathbb{Q}}\left[\Gamma_{T_{n}}^{i} \Gamma_{T_{n+1}}^{j}\left(\sum_{a=1}^{x} D_{r}^{a}-Q\left(i, j, x, T_{n}\right)\right) \mid \sigma\left(Y_{n}\right) \vee \sigma\left(T_{n}\right)\right]=0 .
\end{gathered}
$$

Conversely, let $Q^{\prime}\left(i, j, x, T_{n}\right) \in M_{k+1}$; then, we will prove that, if the process

$$
\mathcal{R}_{0, T_{n}}^{\prime i j}(x)=R_{0, T_{n}}^{i j}(x)-\sum_{r=0}^{n-1} Q^{\prime}\left(i, j, x, T_{r}\right) \Gamma_{T_{r}}^{i} \Gamma_{T_{r+1}}^{j}
$$

is a $\mathbb{G}$-martingale under $\mathbb{Q}$, then the transition probabilities $Q^{\prime}\left(i, j, x, T_{n}\right)$ are equal to $Q\left(i, j, x, T_{n}\right)$.

Since the process $\mathcal{R}_{0, T_{n}}^{\prime i j}(x)$ is a $\mathbb{G}$-martingale under $\mathbb{Q}$, we have that

$$
\mathbb{E}_{\mathbb{Q}}\left(\mathcal{R}_{0, T_{n+1}}^{\prime i j}(x)-\mathcal{R}_{0, T_{n}}^{\prime i j}(x) \mid \mathcal{G}_{T_{n}}\right)=0 .
$$


Consequently,

$$
\begin{gathered}
\mathbb{E}_{\mathbb{Q}}\left(\sum_{a=1}^{x} \sum_{r=0}^{n} \Gamma_{T_{r}}^{i} \Gamma_{T_{r+1}}^{j} D_{r}^{a}-\sum_{r=0}^{n} Q^{\prime}\left(i, j, x, T_{r}\right) \Gamma_{T_{r}}^{i} \Gamma_{T_{r+1}}^{j}\right. \\
\left.-\sum_{a=1}^{x} \sum_{r=0}^{n-1} \Gamma_{T_{r}}^{i} \Gamma_{T_{r+1}}^{j} D_{r}^{a}+\sum_{r=0}^{n-1} Q^{\prime}\left(i, j, x, T_{r}\right) \Gamma_{T_{r}}^{i} \Gamma_{T_{r+1}}^{j} \mid \mathcal{G}_{T_{n}}\right) \\
=\mathbb{E}_{\mathbb{Q}}\left[\Gamma_{T_{n}}^{i} \Gamma_{T_{n+1}}^{j}\left(\sum_{a=1}^{x} D_{r}^{a}-Q^{\prime}\left(i, j, x, T_{n}\right)\right) \mid \mathcal{G}_{T_{n}}\right] \\
=\mathbb{E}_{\mathbb{Q}}\left[\Gamma_{T_{n}}^{i} \Gamma_{T_{n+1}}^{j}\left(\sum_{a=1}^{x} D_{r}^{a}-Q^{\prime}\left(i, j, x, T_{n}\right)\right) \mid \sigma\left(Y_{n}\right) \vee \sigma\left(T_{n}\right)\right]=0 .
\end{gathered}
$$

From (35) and (36), we get that

$$
\mathbb{E}_{\mathbb{Q}}\left[\Gamma_{T_{n}}^{i} \Gamma_{T_{n+1}}^{j}\left(Q\left(i, j, x, T_{n}\right)-Q^{\prime}\left(i, j, x, T_{n}\right)\right) \mid \sigma\left(Y_{n}\right) \vee \sigma\left(T_{n}\right)\right]=0,
$$

from which we get that

$$
Q^{\prime}\left(i, j, x, T_{n}\right)=Q\left(i, j, x, T_{n}\right)
$$

\section{New Closed Analytic Functional Relationships between Forward and Real World Probabilities of Transition}

We will start by altering the conditions of Theorem 1, in a way in which the theorem will still be valid under the new conditions. The new conditions are more restrictive but still remain quite general. However, they will be useful in what follows, in order to provide interesting and simplified closed analytic relations, between the forward probability measure transition probabilities sequences and the corresponding real world probability measure ones.

Note that the conditions under which theorem 1 is valid were the following:

(a) The random variable $\theta_{t}^{-1} \theta_{t+1}$ be $\left(\sigma\left(X_{t}\right) V \sigma\left(S_{t+1}\right)\right)$-measurable for any $t=0,1, \ldots$, $T-1$. As a result, we get that, for every such $t$, we have

$$
\theta_{t}^{-1} \theta_{t+1}=g_{t}\left(X_{t}, S_{t+1}\right)
$$

for some function $g_{t}: \mathbb{K} \times \mathbb{K} \rightarrow \mathbb{R}$.

(b) The random variable $\theta_{t+1}^{-1} \theta_{t+\omega_{X_{t}, S_{t+1}}}$ is $\left(\sigma\left(X_{t}\right) V \sigma\left(S_{t+1}\right) V \sigma\left(\omega_{X_{t}, S_{t+1}}\right)\right)$-measurable for all $t=0,1, \ldots, T-1$. Equivalently,

$$
\theta_{t+1}^{-1} \theta_{t+\omega_{X_{t}, S_{t+1}}}=f_{t+1}\left(X_{t}, S_{t+1}, \omega_{X_{t}, S_{t+1}}\right)
$$

for some function $f_{t+1}: \mathbb{K} \times \mathbb{K} \times \mathbb{N}^{*} \rightarrow \mathbb{R}$.

Let us now assume that the following Condition 1 holds:

Condition 1. (a) The random variable $\theta_{t}^{-1} \theta_{t+1}$ is $\left(\sigma\left(X_{t}\right) V \sigma\left(S_{t+1}\right)\right)$-measurable for any $t=$ $0,1, \ldots, T-1$, and there exists a finite set, denoted by $\mathbb{A}$, such that the random variable $\theta_{t}^{-1} \theta_{t+1}$ admits the following representation:

$$
\theta_{t}^{-1} \theta_{t+1}=1+\sum_{a \in \mathbb{A}} \widehat{g}_{t}^{a}\left(X_{t}\right) \widetilde{g}_{t+1}^{a}\left(X_{t}, S_{t+1}\right)
$$

for some functions $\widehat{g}_{t}^{a}: \mathbb{K} \rightarrow \mathbb{R}$, and $\widetilde{g}_{t+1}^{a}: \mathbb{K} \times \mathbb{K} \rightarrow \mathbb{R}$. (b) The random variable $\theta_{t}^{-1} \theta_{t+\omega_{X_{t}, S_{t+1}}}$ is $\left(\sigma\left(X_{t}\right) V \sigma\left(S_{t+1}\right) V \sigma\left(\omega_{X_{t}, S_{t+1}}\right)\right)$-measurable for every $t=0,1, \ldots, T-1$ and there exists a 
finite set, denoted $\mathbb{B}$, such that the random variable $\theta_{t}^{-1} \theta_{t+\omega_{X_{t}}, S_{t+1}(t)}$ admits the following representation:

$$
\theta_{t+1}^{-1} \theta_{t+\omega_{X_{t}}, S_{t+1}}=1+\sum_{b \in \mathbb{B}} \widehat{f}_{t+1}\left(X_{t}, S_{t+1}\right) \widetilde{f}_{t+1}\left(X_{t}, S_{t+1}, \omega_{X_{t}, S_{t+1}}\right)
$$

for any $\widehat{f}_{t+1}: \mathbb{K} \times \mathbb{K} \rightarrow \mathbb{R}$ and $\widetilde{f}_{t+1}: \mathbb{K} \times \mathbb{K} \times \mathbb{N}^{*}$.

Remark 2. (i) It is apparent that the conditions of Theorem 1 are more general, in the sense that, when Condition 1 holds, then also that of Theorem 1 is satisfied. Conversely, the conditions of Theorem 1 could hold without the Condition 1 to be true. However, Condition 1 is quite general and the assumptions one has to make, during the calibration of the model (Vasileiou and Vassiliou [3]), are such that one would rather freely state that the two conditions are "almost" equivalent for our purposes. (ii) It is known (Vasileiou and Vassiliou [3]) that a discrete-time $\mathbb{G}$-homogeneous Markov chain is a discrete -time $\mathbb{G}$ - inhomogeneous semi-Markov process for which

$$
\begin{gathered}
p_{i j}(t)=p_{i j} \quad \text { for all } i, j \in \mathbb{K} \text { and for all } t=0,1,2, \ldots, \\
h_{i j}(t, 1)=h_{i j}(1)=1 \quad \text { for all } t=0,1,2, \ldots, \\
h_{i j}(t, m)=0 \quad \text { for } m>1 \text { and for all } t=0,1,2, \ldots
\end{gathered}
$$

It is an immediate consequence then to check that, in the case of the $\mathbb{G}$-homogeneous Markov chain model, Condition 1 coincides with Condition (B.2) in Bielecki and Rutkowski [28].

Theorem 5. Let $\left\{X_{t}, S_{t+1}\right\}_{t=0}^{\infty}$ be the $\mathbb{G}$-inhomogeneous semi-Markov process under $\mathbb{Q}$. Suppose that Condition 1 holds. Then, we may choose as (a) of Condition 1

$$
\theta_{t}^{-1} \theta_{t+1}=1+\sum_{v=1}^{k+1} \sum_{l=1}^{k+1} \widehat{g}_{t}^{v l}\left(X_{t}\right) \Delta \mathfrak{C}_{0, t}^{v l}
$$

then any chosen function $\hat{g}_{t}^{i j}(i)$ should satisfy

$$
\frac{1}{p_{i j}(t)}+\frac{\sum_{l \neq j}^{k+1} \widehat{g}_{t}^{i l}(i) p_{i l}(t)}{1-p_{i j}(t)} \geq \hat{g}_{t}^{i j}(i)>\frac{\sum_{l \neq j}^{k+1} \widehat{g}_{t}^{i l}(i) p_{i l}(t)-1}{1-p_{i j}(t)} .
$$

In addition, we may choose as (b) of Condition 1

$$
\hat{\theta}_{t+1}^{-1} \hat{\theta}_{t+m}=1+\sum_{\omega=1}^{m} \sum_{v=1}^{k+1} \sum_{l=1}^{k+1} \widehat{f}_{t+1}^{v l}\left(X_{t}, S_{t+1}\right) \Delta \mathfrak{Y}_{0, t+\omega-1}^{v l}(\varpi),
$$

then any chosen function $\hat{f}_{t+1}^{i j}(i, j)$ should satisfy

$$
\frac{1-h_{i j}(t, m)}{h_{i j}(t, m)\left(1-\sum_{\omega=1}^{m} h_{i j}(t, \omega)\right)} \geq \widehat{f}_{t+1}^{i j}(i, j)>\frac{1}{\sum_{\omega=1}^{m} h_{i j}(t, \omega)-1} .
$$

Then, $\left\{X_{t}, S_{t+1}\right\}_{t=0}^{\infty}$ is a discrete time $\mathbb{G}$-inhomogeneous semi-Markov process under the forward probability measure $\mathbb{Q}_{T}$, and, for every $t=0,1,2, \ldots, T-1$, we have the following:

$$
\begin{gathered}
\text { (a) } \theta_{t}=1+\sum_{u=1}^{t} \theta_{u-1} \sum_{v=1}^{k+1} \sum_{l=1}^{k+1} \widehat{g}_{u}^{v l}\left(X_{u}\right) \widetilde{g}_{u+1}^{v l}\left(X_{u}, S_{u+1}\right) . \\
(\mathbf{b})_{F} p_{i j}(t)=p_{i j}(t)\left(1+\hat{g}_{t}^{i j}(i)-\sum_{l=1}^{k+1} p_{i l}(t) \widehat{g}_{t}^{i l}(i)\right) . \\
\text { (c) } F h_{i j}(t, r)=h_{i j}(t, r)\left(1+\widehat{f}_{t+1}^{i j}(i, j)-\sum_{m=1}^{r} \widehat{f}_{t+1}^{i j}(i, j) h_{i j}(t-m+1, m)\right) .
\end{gathered}
$$




$$
\text { (d) } \hat{\theta}_{t}=1+\sum_{u=1}^{t-1} \hat{\theta}_{u}\left(\sum_{\omega=1}^{2} \sum_{v=1}^{k+1} \sum_{l=1}^{k+1} \widehat{f}_{t+1}^{v l}(i, j) \Delta \mathfrak{Y}_{0, t+\omega-2}^{v l}(\boldsymbol{\omega})\right) .
$$

In addition, the chosen functions $\hat{g}_{t}^{i j}(i)$ and $\hat{f}_{t+1}^{i j}(i, j)$ should be such that $\hat{\theta}_{t}=\theta_{t+1}$.

Proof. In order for Condition 1 be acceptable, it should satisfy two prerequisites. First, $\theta_{t}$ should be a martingale process adapted to $\mathcal{G}_{t}$ and secondly $\theta_{t}$ should be strictly positive. From Equation (25) and Theorem 5, we have

$$
\begin{gathered}
\Delta \mathfrak{C}_{0, t}^{v l}=\mathfrak{C}_{0, t+1}^{v l}-\mathfrak{C}_{0, t}^{v l}=\sum_{u=0}^{t} H_{u}^{v} \mathrm{Y}_{u+1}^{l}-\sum_{u=0}^{t} p_{v l}(u) H_{u}^{v}-\sum_{u=0}^{t-1} H_{u}^{v} \mathrm{Y}_{u+1}^{l}+\sum_{u=0}^{t-1} p_{v l}(u) H_{u}^{v} \\
=H_{t}^{v} \mathrm{Y}_{t+1}^{l}-p_{v l}(t) H_{t}^{v}=\mathbf{1}_{\left\{X_{t}=v, S_{t+1}=l\right\}}-p_{v l}(t) \mathbf{1}_{\left\{X_{t}=v\right\}}
\end{gathered}
$$

and so the process $\Delta \mathfrak{C}_{0, t}^{v l}$ is $\sigma\left(X_{t}\right) V \sigma\left(S_{t+1}\right)$-measurable, i.e., it is a function; let us say $\widetilde{g}_{t+1}^{v l}\left(X_{t}, S_{t+1}\right)$, where

$$
\widetilde{g}_{t+1}^{v l}(i, j)=\mathbf{1}_{\left\{X_{t}=v\right\}}(i)\left\{\mathbf{1}_{\left\{S_{t+1}=l\right\}}(j)-p_{v l}(t)\right\}
$$

Now, we have

$$
\begin{gathered}
\mathbb{E}_{\mathbb{Q}}\left(\sum_{v=1}^{k+1} \sum_{l=1}^{k+1} \widehat{g}_{t}^{v l}\left(X_{t}\right) \Delta \mathfrak{C}_{0, t}^{v l} \mid \mathcal{G}_{t}\right)=\cdot \sum_{v=1}^{k+1} \sum_{l=1}^{k+1} \widehat{g}_{t}^{v l}\left(X_{t}\right) \mathbb{E}_{\mathbb{Q}}\left(\Delta \mathfrak{C}_{0, t}^{v l} \mid \mathcal{G}_{t}\right) \\
=\sum_{v=1}^{k+1} \sum_{l=1}^{k+1} \widehat{g}_{t}^{v l}\left(X_{t}\right) \mathbb{E}_{\mathbb{Q}}\left(H_{t}^{v} Y_{t+1}^{l}-p_{v l}(t) H_{t}^{v} \mid \mathcal{G}_{t}\right) \\
=\sum_{v=1}^{k+1} \sum_{l=1}^{k+1} \widehat{g}_{t}^{v l}\left(X_{t}\right) \mathbb{E}_{\mathbb{Q}}\left(H_{t}^{v} Y_{t+1}^{l}-p_{v l}(t) H_{t}^{v} \mid X_{t}=v\right)=0
\end{gathered}
$$

From Equation (38), it is apparent that we may choose as $(a)$ of Condition 4.1 the following:

$$
\theta_{t}^{-1} \theta_{t+1}=1+\sum_{v=1}^{k+1} \sum_{l=1}^{k+1} \widehat{g}_{t}^{v l}\left(X_{t}\right) \Delta \mathfrak{C}_{0, t}^{v l}
$$

Then, from (41), we get

$$
\mathbb{E}_{\mathbb{Q}}\left(\theta_{t}^{-1} \theta_{t+1} \mid \mathcal{G}_{t}\right)=(\text { due to }(40))=1,
$$

and consequently

$$
\mathbb{E}_{\mathbb{Q}}\left(\theta_{t+1} \mid \mathcal{G}_{t}\right)=\theta_{t} .
$$

Thus, the stochastic process $\theta_{t}$ as defined by (41) is a martingale under $\mathbb{Q}$. In addition, $\theta_{t}$ as defined by (41) should be strictly positive. It is easy to check that it is sufficient to have that

$$
\sum_{v=1}^{k+1} \sum_{l=1}^{k+1} \widehat{g}_{t}^{v l}\left(X_{t}\right) \Delta \mathfrak{C}_{0, t}^{v l}>-1 .
$$

Now, from (43) for any $i, j \in \mathbb{K}$, we equivalently have

$$
\sum_{v=1}^{k+1} \sum_{l=1}^{k+1} \widehat{g}_{t}^{v l}(i) \mathbf{1}_{\left\{X_{t}=v\right\}}(i)\left\{\mathbf{1}_{\left\{S_{t+1}=l\right\}}-p_{v l}(t)\right\}>-1,
$$

or, equivalently

$$
\sum_{l=1}^{k+1} \hat{g}_{t}^{i l}(i)\left\{\mathbf{1}_{\left\{S_{t+1}=l\right\}}-p_{v l}(t)\right\}>-1,
$$


from which we get

$$
\hat{g}_{t}^{i j}(i)-\sum_{l=1}^{k+1} \hat{g}_{t}^{i l}(i) p_{i l}(t)>-1,
$$

and finally

$$
\widehat{g}_{t}^{i j}(i)>\frac{\sum_{l \neq j}^{k+1} \widehat{g}_{t}^{i l}(i) p_{i l}(t)-1}{1-p_{i j}(t)}
$$

Thus, any chosen function $\hat{g}_{t}^{i j}(i)$ should satisfy (44).

From Equation (41), since $\theta_{0}=1$, the process $\theta_{t}$ is given by

$$
\theta_{t}=1+\sum_{u=1}^{t} \theta_{u-1} \sum_{v=1}^{k+1} \sum_{l=1}^{k+1} \widehat{g}_{u-1}^{v l}\left(X_{u-1}\right) \Delta \mathfrak{C}_{0, u-1}^{v l}
$$

Since Condition 1 holds, then the conditions of Theorem 5 are valid and thus we have

$$
\begin{gathered}
F_{F} p_{i j}(t)=p_{i j}(t)\left(1+\sum_{v=1}^{k+1} \sum_{l=1}^{k+1} \widehat{g}_{t}^{\nu l}(i) \mathbf{1}_{\left\{X_{t}=\nu\right\}}(i)\left\{\mathbf{1}_{\left\{S_{t+1}=l\right\}}(j)-p_{v l}(t)\right\}\right) \\
=p_{i j}(t)\left(1+\sum_{l=1}^{k+1} \widehat{g}_{t}^{i l}(i)\left\{\mathbf{1}_{\left\{S_{t+1}=l\right\}}(j)-p_{i l}(t)\right\}\right) \\
=p_{i j}(t)\left(1+\widehat{g}_{t}^{i j}(i)-\sum_{l=1}^{k+1} p_{i l}(t) \widehat{g}_{t}^{i l}(i)\right) .
\end{gathered}
$$

From Equation (46), it is apparent that the choice of any function $\hat{g}_{t}^{i j}(i)$, apart from satisfying (44), which guarantees the positivity of $F p_{i j}(t)$, should be such that

$$
p_{i j}(t)\left(1+\hat{g}_{t}^{i j}(i)-\sum_{l=1}^{k+1} p_{i l}(t) \widehat{g}_{t}^{i l}(i)\right) \leq 1
$$

from which we get that

$$
\widehat{g}_{t}^{i j}(i) \leq \frac{1}{p_{i j}(t)}+\frac{\sum_{l \neq j}^{k+1} \widehat{g}_{t}^{i l}(i) p_{i l}(t)}{1-p_{i j}(t)} .
$$

Now, from Theorem 5, we have

$$
\begin{gathered}
\Delta \mathfrak{Y}_{0, t+m-1}^{v l}(m)=\mathfrak{Y}_{0, t+m}^{v l}(m)-\mathfrak{Y}_{0, t+m-1}^{v l}(m) \\
=Y_{0, t+m}^{v l}(m)-\sum_{u=0}^{t} h_{v l}(u, m) \Phi_{u+1}^{v l}-Y_{0, t+m-1}^{v l}(m)+\sum_{u=0}^{t-1} h_{v l}(u, m) \Phi_{u+1}^{v l} \\
=\sum_{u=0}^{t} \Phi_{u+1}^{v l} H_{u+m}^{l}-\sum_{u=0}^{t} h_{v l}(u, m) \Phi_{u+1}^{v l}-\sum_{u=0}^{t-1} \Phi_{u+1}^{v l} H_{u+m}^{l}+\sum_{u=0}^{t-1} h_{v l}(u, m) \Phi_{u+1}^{v l} \\
=H_{t}^{v l} Y_{t+1}^{l}\left(H_{t+m}^{l}-h_{v l}(t, m)\right) \\
=\mathbf{1}_{\left\{X_{t}=v, S_{t+1}=l\right\}}\left\{\mathbf{1}_{\left\{\omega_{v l}(t)=m\right\}}-h_{v l}(t, m)\right\} .
\end{gathered}
$$

and so the process $\Delta \mathfrak{Y}_{0, t+m-1}^{v l}(m)$ is a function of the form

$$
\widetilde{f}_{t+1}\left(X_{t}, S_{t+1}, \omega_{X_{t}, S_{t+1}}(t)\right)
$$


where

$$
\widetilde{f}_{t+1}(i, j, r)=\mathbf{1}_{\left\{X_{t}=v, S_{t+1}=l\right\}}(i, j)\left\{\mathbf{1}_{\left\{\omega_{v l}(t)=m\right\}}(r)-h_{v l}(t, r)\right\} .
$$

It is apparent, from Equations (48) and (49), that we may choose part b) of Condition 1 to be of the following form:

$$
\hat{\theta}_{t+1}^{-1} \hat{\theta}_{t+m}=1+\sum_{\omega=1}^{m} \sum_{v=1}^{k+1} \sum_{l=1}^{k+1} \widehat{f}_{t+1}^{v l}\left(X_{t}, S_{t+1}\right) \Delta \mathfrak{Y}_{0, t+\omega-1}^{v l}(๗) .
$$

Now, we have

$$
\begin{gathered}
\mathbb{E}_{\mathbb{Q}}\left(\hat{\theta}_{t+1}^{-1} \hat{\theta}_{t+m} \mid \mathcal{G}_{t+1}\right)=1+\sum_{\omega=1}^{m} \sum_{v=1}^{k+1} \sum_{l=1}^{k+1} \widehat{f}_{t+1}^{v l}\left(X_{t}, S_{t+1}\right) \mathbb{E}_{\mathbb{Q}}\left(\Delta \mathfrak{Y}_{0, t+\omega-1}^{v l}(\omega) \mid \mathcal{G}_{t+1}\right) \\
=1+\sum_{\omega=1}^{m} \sum_{v=1}^{k+1} \sum_{l=1}^{k+1} \widehat{f}_{t+1}^{v l}\left(X_{t}, S_{t+1}\right) \mathbb{E}_{\mathbb{Q}}\left(H_{t}^{v} Y_{t+1}^{l}\left(H_{t+\infty}^{l}-h_{v l}(t, \omega)\right) \mid \mathcal{G}_{t+1}\right) \\
=1+\sum_{\omega=1}^{m} \sum_{v=1}^{k+1} \sum_{l=1}^{k+1} \widehat{f}_{t+1}^{v l}\left(X_{t}, S_{t+1}\right) \\
\times \mathbb{E}_{\mathbb{Q}}\left(H_{t}^{v} Y_{t+1}^{l}\left(H_{t+\infty}^{l}-h_{v l}(t, \omega)\right) \mid X_{t}=v, S_{t+1}=l\right)=1
\end{gathered}
$$

Thus, we get that

$$
\mathbb{E}_{\mathbb{Q}}\left(\hat{\theta}_{t+m} \mid \mathcal{G}_{t+1}\right)=\hat{\theta}_{t+1}
$$

and consequently the process $\hat{\theta}_{t}$ is a martingale under $\mathbb{Q}$. In addition, $\hat{\theta}_{t}$ as defined by (50) should be strictly positive. It is easy to check that it is sufficient to have

$$
\sum_{\omega=1}^{m} \sum_{v=1}^{k+1} \sum_{l=1}^{k+1} \widehat{f}_{t+1}^{v l}\left(X_{t}, S_{t+1}\right) \Delta \mathfrak{Y}_{0, t+\infty-1}^{v l}(\omega)>-1 .
$$

For any $i, j, r$, it is equivalent to have

$$
\begin{gathered}
1+\sum_{\omega=1}^{m} \sum_{v=1}^{k+1} \sum_{l=1}^{k+1} \widehat{f}_{t+1}^{v l}(i, j) \mathbf{1}_{\left\{X_{t}=v, S_{t+1}=l\right\}}(i, j)\left\{\mathbf{1}_{\left\{\omega_{v l}(t)=\omega\right\}}(r)-h_{v l}(t, \omega)\right\} \\
=1+\sum_{\omega=1}^{m} \widehat{f}_{t+1}^{i j}(i, j)\left\{\mathbf{1}_{\left\{\omega_{i j}(t)=\omega\right\}}(r)-h_{i j}(t, \omega)\right\} \\
=1+\widehat{f}_{t+1}^{i j}(i, j)-\sum_{\omega=1}^{m} \hat{f}_{t+1}^{i j}(i, j) h_{i j}(t, \omega)>0 .
\end{gathered}
$$

Thus, the choice of $\hat{f}_{t+1}^{i j}(i, j)$ should be such that

$$
\widehat{f}_{t+1}^{i j}(i, j)>\frac{1}{\sum_{\omega=1}^{m} h_{i j}(t, \omega)-1}
$$

Since Condition 1 holds, then the conditions of Theorem 5 are valid and thus we have

$$
\begin{aligned}
{ }_{F} h_{i j}(t, m)=h_{i j}(t, m) & \left(1+\sum_{\omega=1}^{m} \sum_{v=1}^{k+1} \sum_{l=1}^{k+1} \widehat{f}_{t+1}^{v l}(i, j) \Delta \mathfrak{Y}_{0, t+\omega-1}^{v l}(\boldsymbol{\omega})\right) \\
=h_{i j}(t, m) & \left(1+\sum_{\omega=1}^{m} \sum_{v=1}^{k+1} \sum_{l=1}^{k+1} \widehat{f}_{t+1}^{l l}(i, j) \mathbf{1}_{\left\{X_{t}=v, S_{t+1}=l\right\}}(i, j)\right. \\
& \left.\times\left\{\mathbf{1}_{\left\{\omega_{v l}(t)=m\right\}}(r)-h_{v l}(t, \omega)\right\}\right)
\end{aligned}
$$




$$
\begin{gathered}
=h_{i j}(t, m)\left(1+\sum_{\omega=1}^{m} \hat{f}_{t+1}^{i j}(i, j)\left\{\mathbf{1}_{\left\{\omega_{i j}(t)=m\right\}}(r)-h_{i j}(t, \omega)\right\}\right) \\
=h_{i j}(t, m)\left(1+\widehat{f}_{t+1}^{i j}(i, j)-\sum_{\omega=1}^{m} \widehat{f}_{t+1}^{i j}(i, j) h_{i j}(t, \omega)\right) .
\end{gathered}
$$

From Equation (55), it is apparent that any choice of a function $\hat{f}_{t+1}^{i j}(i, j)$ apart from satisfying Equation (23) should also satisfy the following relation:

$$
h_{i j}(t, m)\left(1+\hat{f}_{t+1}^{i j}(i, j)-\sum_{\omega=1}^{m} \hat{f}_{t+1}^{i j}(i, j) h_{i j}(t, \mathfrak{\omega})\right) \leq 1,
$$

which is equivalent with

$$
\widehat{f}_{t+1}^{j}(i, j) \leq \frac{1-h_{i j}(t, m)}{h_{i j}(t, m)\left(1-\sum_{\omega=1}^{m} h_{i j}(t, \omega)\right)} .
$$

From Equation (50), since the initial information is included in the interval $[0,1)$, we may assume that $\hat{\theta}_{1}=1$ and then we get

$$
\hat{\theta}_{t}=1+\sum_{u=1}^{t-1} \hat{\theta}_{u}\left(\sum_{\omega=1}^{2} \sum_{v=1}^{k+1} \sum_{l=1}^{k+1} \widehat{f}_{u}^{v l}(i, j) \Delta \mathfrak{Y}_{0, u+\infty-2}^{v l}(๗)\right) .
$$

It is apparent that the choices of $\hat{g}_{t}^{i j}(i)$ and $\hat{f}_{t+1}^{i j}(i, j)$, in addition to the already created restrictions, should also be such that

$$
\theta_{t+1}=\hat{\theta}_{t} \text { for every } t=0,1, \ldots, T .
$$

\section{Model Calibration}

The problem of calibration of the non-homogeneous semi-Markov model is an important one and will be resolved in the present section. The calibration firstly was discussed by Vasileiou and Vassiliou [3] in Section 6. In the present, we will resolve the calibration of the semi-Markov model under the new assumptions and the algorithm provided below. Although similar in nature and perception to the algorithm in Section 6 of Vasileiou and Vassiliou [3], it also serves the purpose of clarifying many details. In addition, by understanding in some depth the present Algorithm 8.1, we could go back in the algorithm in Section 6 of Vasileiou and Vassiliou [3] and easily make some needed corrections.

Available Data. Allow that $T^{*}$ is the present time. We assume that the available data span are for the time window $\left[0, T^{*}\right]$. These data will consist of the following type:

(i) From historical data on credit migrations, we firstly estimate the matrix sequences $\{\tilde{\mathbf{P}}(t)\}_{t=0}^{T^{*}-1}$ and $\{\tilde{\mathbf{H}}(t, m)\}_{t, m=0}^{T^{*}-1}$ during our time window $\left[0, T^{*}\right]$. The main problem in estimation was that some issuers had at some time during the time window withdrawn from the rating process. This flow was treated as type III censoring (see Lee [32]) as in biomedical data. Equivalently, it is also met as right censoring, in other studies, see for example McClean and Gribbin [33,34]. It is inherent that it is also assumed that the reason for the rating being withdrawn is not the understanding of possibility of a default. In the semi-Markov chain case, it is necessary to estimate the conditional density of duration in each grade and the duration in a grade before default. The same problem appears in manpower planning, where people move among the grades of an organization. For more details, see McClean et al. [35,36]. Given the above peculiarities of the data available and models used, the estimation methodology appeared in Subsection 6.1 of Vasileiou 
and Vassiliou [3]: (ii) The observed values of $B(t, T)$, for every $t=0,1, \ldots, T^{*}$ and $T=t+1, \ldots, T^{*}$.

(iii) The observed values of $D_{i}^{\delta}(t, T)$, for every $i=1,2, \ldots, k, t=1,2, \ldots, T^{*}$, and $T=t+1, \ldots, T^{*}$.

(iv) Observed values on recovery rates, $\delta$, in the time window $\left[0, T^{*}\right]$.

Our target is to estimate the sequences $\left\{{ }_{F} \tilde{\mathbf{P}}(t)\right\}_{t=0}^{T^{*}-1}$ and $\left\{{ }_{F} \tilde{\mathbf{H}}(t, m)\right\}_{t, m=0}^{T^{*}-1}$, within $\left[0, T^{*}\right]$, that is, the time window. In fact, we do not need to calculate $\left\{{ }_{F} \tilde{\mathbf{P}}(t)\right\}_{t=0}^{T^{*}-1}$ and $\left\{{ }_{F} \tilde{\mathbf{H}}(t, m)\right\}_{t, m=0}^{T^{*}-1}$ separately as it is shown below. What is needed is to find the entrance probabilities $\widetilde{\mathbf{E}}(s, t)$ defined below, which as it is proved in what follows are functions of the Hadamard products ${ }_{F} \mathbf{P}(t) \triangle_{F} \mathbf{H}(t, m)$.

\subsection{The Risk Premium Assumptions and Their Consequences}

In order to calibrate the non-homogeneous semi-Markov chain models that are compatible with Theorem 5, we need to make the following assumptions, which are analogous with the case of the homogeneous Markov model.

Assumption 1. (The risk premium assumptions).

(i) For any $t \in\left[0, T^{*}-1\right]$ and $i, j=1,2, \ldots, k$, assume:

$$
g_{t}^{i j}\left(X_{t}\right)=g_{t}^{i}\left(X_{t}\right)
$$

(ii) For any $t \in\left[0, T^{*}-1\right], i=1,2, \ldots, k$, and $j=1,2, \ldots, k+1$, assume:

$$
f_{t+1}^{i j}\left(X_{t} S_{t+1}\right)=f_{t+1}^{i}\left(X_{t}\right) .
$$

The consequences of the Assumption 8.1 on the results of Theorem 7 are the following:

(a) For all $i=1,2, \ldots, k, j=1,2, \ldots, k+1$ and for every $t \in\left[0, T^{*}-1\right]$

$$
\begin{aligned}
{ }_{F} p_{i j}(t)=p_{i j}(t)\left(1+g_{t}^{i}(i)\right. & \left.-\sum_{l \neq i}^{k+1} p_{i l}(t) g_{t}^{i}(i)\right)=p_{i j}(t)\left(1+p_{i i}(t) g_{t}^{i}(i)\right) \\
& =\widetilde{g}_{t}(i) p_{i j}(t) .
\end{aligned}
$$

(b) For all $i, j=1,2, \ldots, k$ and $j=k+1$ and for every $t \in\left[0, T^{*}-1\right]$,

$$
\begin{gathered}
{ }_{F} h_{i j}(t, r)=h_{i j}(t, r)\left(1+f_{t+1}^{i}(i)\left[1-\sum_{m=1}^{r} h_{i j}(t-m+1, m)\right]\right) \\
=\widetilde{f}_{t}(i, r) h_{i j}(t, r) .
\end{gathered}
$$

\subsection{The Real and Forward Entrance Probability Measures}

Allow the following real probability measures which we call the entrance probabilities for the non-homogeneous semi-Markov chain:

$\mathbb{Q}\{$ the bond enters credit class $j$ at time $t \mid$ it entered credit class $i$ at time $s\}$

$$
=\mathbb{Q}\left(X_{t}=j \mid X_{s}=i\right)=e_{i j}(s, t) .
$$

and $\mathbf{E}(s, t)=\left\{e_{i j}(s, t)\right\}, i, j=1,2, \ldots, k$. In addition, let $\widetilde{\mathbf{E}}(s, t)$ be the matrices of entrance probabilities such that

$$
\widetilde{\mathbf{E}}(s, t)=\left(\begin{array}{cc}
\mathbf{E}(s, t) & \mathbf{e}_{k+1}^{\top}(s, t) \\
\mathbf{0} & \mathbf{1}_{\{t-s=1\}}
\end{array}\right),
$$


where $\mathbf{e}_{k+1}^{\top}(s, t)=\left[e_{1 k+1}(s, t), e_{2 k+1}(s, t), \ldots, e_{k k+1}(s, t)\right]^{\top}$, with

$$
e_{i k+1}(s, t)=\mathbb{Q}\left(X_{t}=k+1 \mid X_{s}=i\right) \text { for } i=1,2, \ldots, k .
$$

From Vasileiou and Vassiliou [3] p.182, we get

$$
\mathbf{E}(s, t)=\sum_{m=0}^{t-s}[\mathbf{P}(s) \triangle \mathbf{H}(s, m)] \mathbf{E}(s+m, t) \text { for } s<t,
$$

with $\mathbf{E}(s, s)=\mathbf{I}$ for $s=0,1, \ldots$, and

$$
\mathbf{e}_{k+1}^{\top}(s, t)=\sum_{m=0}^{t-s}[\mathbf{P}(s) \triangle \mathbf{H}(s, m)] \mathbf{e}_{k+1}^{\top}(s+m, t)+\mathbf{p}_{k+1}^{\top}(s) \triangle \mathbf{h}_{k+1}^{\top}(s, t-s),
$$

with $\mathbf{e}_{k+1}^{\top}(s, s)=0$ for $s=0,1, \ldots T^{*}-1$.

From Theorem 1, we know that the change to forward measure preserves the semiMarkov structure, hence

$$
\begin{aligned}
\mathbb{Q}_{T}\left\{\tau>T \mid X_{t}=i\right\}= & 1-\mathbb{Q}_{T}\left\{\tau \leq T \mid X_{t}=i\right\}= \\
& 1-\sum_{s=t}^{T} F e_{i, k+1}(s, T) .
\end{aligned}
$$

Using (9) and (67), we get

$$
{ }_{F} \mathbf{e}_{k+1}^{\top}(t, t+1)=\frac{B(t, t+1)-D_{i}^{\delta}(t, t+1)}{(1-\delta) B(t, t+1)} \text { for every } t \in\left[0, T^{*}-1\right],
$$

and more generally we have

$$
\frac{B(t, t+n)-D_{i}^{\delta}(t, t+n)}{(1-\delta) B(t, t+n)}=\sum_{k=0}^{n-1}{ }_{F} \mathbf{e}_{k+1}^{\top}(t, t+1) \text { for every } t \in\left[0, T^{*}-1\right] .
$$

\subsection{An Algorithm for Calculating the Forward Entrance Probabilities}

We are now in a position to propose an algorithm for evaluating the forward entrance probabilities which in summary is as follows:

Algorithm 8.1. (Risk Premium Algorithm).

Step 1. For all $t=0,1, \ldots, T^{*}-1$, and $i=1,2, \ldots, k$, using (68), we find

$$
F e_{i, k+1}(t, t+1)=\frac{B(t, t+1)-D_{i}^{\delta}(t, t+1)}{(1-\delta) B(t, t+1) .}
$$

Since the forward measure preserves the semi-Markov structure using relation (66), we get

$$
{ }_{F} \mathbf{e}_{k+1}^{\top}(t, t+1)={ }_{F} \mathbf{p}_{k+1}^{\top}(t) \triangle_{F} \mathbf{h}^{\top}(t, 1),
$$

and, therefore, for all $t=0,1, \ldots, T^{*}-1$, and $i=1,2, \ldots, k$ using (70) and (71), we obtain

$$
\widetilde{g}_{t}(i) \widetilde{f}_{t}(i, 1)=\frac{B(t, t+1)-D_{i}^{\delta}(t, t+1)}{(1-\delta) B(t, t+1) p_{i, k+1}(t) h_{i, k+1}(t, 1)} .
$$

Now, from Theorem 5, we know that the forward measure preserves the semi-Markov process, hence, from (65), we get that

$$
{ }_{F} \mathbf{E}(t, t+1)={ }_{F} \mathbf{P}(t) \triangle_{F} \mathbf{H}(t, 1) ;
$$


therefore, for all $t=0,1, \ldots, T^{*}-1$, we find

$$
{ }_{F} \mathbf{E}(t, t+1)=\left(\begin{array}{ccc}
\tilde{g}_{t}(1) \tilde{f}_{t}(1,1) p_{11}(t) h_{11}(t, 1) & \ldots & \tilde{g}_{t}(1) \tilde{f}_{t}(1,1) p_{1 k}(t) h_{1 k}(t, 1) \\
\ldots & \ldots & \ldots \\
\tilde{g}_{t}(k) \tilde{f}_{t}(k, 1) p_{k 1}(t) h_{k 1}(t, 1) & \ldots & \tilde{g}_{t}(k) \tilde{f}_{t}(k, 1) p_{k 1}(t) h_{k 1}(t, 1)
\end{array}\right),
$$

and finally

$$
{ }_{F} \tilde{\mathbf{E}}(t, t+1)=\left(\begin{array}{cc}
{ }_{F} \mathbf{E}(t, t+1) & { }_{F} \mathbf{e}_{k+1}^{\top}(t, t+1) \\
0 & 1
\end{array}\right) .
$$

Step 2. Using (69) and since, in Step 1 by relation (70), we have evaluated ${ }_{F} e_{i, k+1}(t+1, t+2)$ for all $t$ and all $i$ for $t=0,1, . ., T^{*}-2$, for $i=1,2, \ldots, k$, we obtain

$$
{ }_{F} e_{i, k+1}(t, t+2)=\frac{B(t, t+2)-D_{i}(t, t+2)}{(1-\delta) B(t, t+2)}-{ }_{F} e_{i, k+1}(t+1, t+2)
$$

From (66), since the change to forward measure preserves the semi-Markov process, we find that

$$
{ }_{F} \mathbf{e}_{k+1}(t, t+2)=\left[{ }_{F} \mathbf{P}(t) \triangle_{F} \mathbf{H}(t, 1)\right]_{F} \mathbf{e}_{k+1}^{\top}(t+1, t+2)+{ }_{F} \mathbf{p}_{k+1}^{\top}(t) \triangle_{F} \mathbf{h}_{k+1}^{\top}(t, 2)
$$

Define by $\{\mathbf{B}\}_{i j}$ the $i j$-element of the matrix $\mathbf{B}$, then (77) could be written as

$$
\begin{gathered}
{ }_{F} e_{i, k+1}(t, t+2)=\sum_{l=1}^{k}\left\{\left[{ }_{F} \mathbf{P}(t) \triangle_{F} \mathbf{H}(t, 1)\right]\right\}_{i l F} e_{l, k+1}(t+1, t+2)+ \\
{ }_{F} p_{i, k+1}(t) \triangle_{F} h_{i, k+1}(t, 2),
\end{gathered}
$$

and

$$
\begin{aligned}
{ }_{F} e_{i, k+1}(t, t+2) & =\sum_{l=1}^{k}\left\{\left[{ }_{F} \mathbf{P}(t) \triangle_{F} \mathbf{H}(t, 1)\right]\right\}_{i l F} e_{l, k+1}(t+1, t+2) \\
& +\widetilde{g}_{t}(i) \widetilde{f}_{t}(i, 2) p_{i, k+1}(t) h_{i, k+1}(t, 2)
\end{aligned}
$$

Solve the system (76) and (79) to obtain

$$
\widetilde{g}_{t}(i) \widetilde{f}_{t}(i, 2) \text { for } i=1,2, \ldots, k \text { and } t=0,1, \ldots, T^{*}-1 .
$$

Hence, now we are in a position to evaluate

$$
\begin{gathered}
{ }_{F} \mathbf{P}(t) \triangle_{F} \mathbf{H}(t, t+2)= \\
\left(\begin{array}{ccc}
\tilde{g}_{t}(1) \tilde{f}_{t}(1,2) p_{11}(t) h_{11}(t, 2) & \ldots & \tilde{g}_{t}(1) \tilde{f}_{t}(1,2) p_{1 k}(t) h_{1 k}(t, 2) \\
\ldots & \ldots & \ldots \\
\tilde{g}_{t}(k) \tilde{f}_{t}(k, 2) p_{k 1}(t) h_{k 1}(t, 2) & \ldots & \tilde{g}_{t}(k) \tilde{f}_{t}(k, 2) p_{k 1}(t) h_{k 1}(t, 2)
\end{array}\right)
\end{gathered}
$$
we get

Since the change to forward measure preserves the semi-Markov property from (65),

$$
{ }_{F} \mathbf{E}(t, t+2)=\left[{ }_{F} \mathbf{P}(t) \triangle_{F} \mathbf{H}(t, t+1)\right] \mathbf{E}(t+1, t+2)+{ }_{F} \mathbf{P}(t) \triangle_{F} \mathbf{H}(t, t+2) .
$$

Now, from (82) and using (81) and (75), we evaluate for $t=0,1, \ldots, T^{*}-2$ the entrance probabilities ${ }_{F} \mathbf{E}(t, t+2)$. Hence, Step 2 is concluded by collecting or the evaluations done in the matrices:

$$
{ }_{F} \tilde{\mathbf{E}}(t, t+1)=\left(\begin{array}{cc}
{ }_{F} \mathbf{E}(t, t+1) & { }_{F} \mathbf{e}_{k+1}^{\top}(t, t+1) \\
0 & 1
\end{array}\right) .
$$


We repeat the methodology indicated by Step 2 for evaluating all desired values of ${ }_{F} \tilde{\mathbf{E}}(t, t+n)$ for $n=1,2, \ldots, T^{*}-t$.

If we wish to find separate values of $\widetilde{g}_{t}(i)$ and $\widetilde{f}_{t}(i, m)$ for our time window $\left[0, T^{*}\right]$, do as follows. Let, for every specific $t, M_{i j}(t)$ to be the maximum of $\mathrm{m}$ with $h_{i j}(t, m)$ strictly positive, and define

$$
h_{i j}\left(t, M_{i j}(t)\right)=1-\sum_{m=1}^{M_{i j}(t)-1} h_{i j}(t, m) .
$$

From Theorem 5, the semi-Markov structure is preserved therefore

$$
\sum_{m=1}^{\infty}{ }_{F} h_{i j}(t, m)=1 \text { for every } t \in\left[0, T^{*}-1\right] \text { and } i, j=1,2, \ldots, k,
$$

from which we get

$$
\sum_{m=1}^{M_{i j}(t)} \widetilde{f}_{t}(i, m) h_{i j}(t, m)=1,
$$

and consequently

$$
\widetilde{g}_{t}(i)=\sum_{m=1}^{M_{i j}(t)} \widetilde{g}_{t}(i) \widetilde{f}_{t}(i, m) h_{i j}(t, m),
$$

which provides the values $\widetilde{g}_{t}^{i}(i)$, and, consequently, the corresponding values

$$
\widetilde{f}_{t}(i, 1), \widetilde{f}_{t}(i, 2), \ldots, \widetilde{f}_{t}\left(i, M_{i j}(t)\right) .
$$

Now, from (60) and (61), we get that

$$
g_{t}^{i}(i)=\frac{\widetilde{g}_{t}(i)-1}{p_{i i}(t)}
$$

and

$$
f_{t+1}^{i j}(i)=\frac{\widetilde{f}_{t}(i, m)-1}{1-\sum_{m=1}^{r} h_{i j}(t-m+1, m)} .
$$

\section{An Illustrative Application}

In this section, we provide an illustrative application of the non-homogeneous semiMarkov chain in the evolution of migration process in credit risk. Credit rating classes are typically identified by a finite set of elements, which we call the set of credit rates. A good account of the rating systems of these agencies could be found in [37]. Most rating systems involve both quantitative and qualitative information. In our semi-Markov model, the set of states are the credit grades, which, in many studies, are identified as

$$
\{\text { Aaa, Aa, A, Baa, Ba, B, Caa, default }\} \text {. }
$$

Following the Standard and Poor's definitions, the above rating categories have the following meaning:

Aaa : A bond or an obligation is rated Aaa declaring that the obligor's capacity to meet its financial commitment on the obligation is extremely strong.

Aa : A bond or an obligation is rated Aa when it differs from the highest rated obligations only by a small degree. The obligor's capacity to meet its financial commitment on the obligation is very strong.

A : A bond or an obligation is rated A when it is more susceptible to the adverse changes in the economic conditions. The obligor's capacity to meet its financial commitment on the obligation is still strong. 
Baa : A bond or an obligation rated Baa exhibits sufficient protection. However, under adverse changes in the economic conditions, its capacity to meet its obligations will probably be weakened more.

Ba : A bond or an obligation rated Baa is considered to be a speculative issue. However, it is less vulnerable than other speculative issues.

B : A bond or an obligation rated B is more vulnerable to nonpayment than obligations rated Ba. However, currently, the obligor has the capacity to meet payments. Adverse changes in economic conditions most probably will impair that capacity.

Caa : A bond or an obligation rated Caa is currently highly vulnerable to nonpayment.

Therefore, there are seven grades and the default grade. In the present application for size purposes of the respective matrices, we will merge the grades into three and the default. If we do not do that, then we will have to provide a large number of $8 \times 8$ matrices in the present application, which will take too much space. That is, the state space will be $S=\{1,2,3,4\}$, where 4 is the default state, 1 is the grade with least risk involved and 3 the grade with the maximum risk involved. Our time window is $[0,3]$. The observed values of $B(t, T)$ are provided by the market and for our present time window are the following:

Default free bonds for the time window $[0,3]$.

$$
\begin{aligned}
& B(0,0)=1 . \\
& B(0,1)=0.9, B(1,1)=1 . \\
& B(0,2)=0.87, B(1,2)=0.888, B(2,2)=1 . \\
& B(0,3)=0.8, B(1,3)=0.84, B(2,3)=0.86, B(3,3)=1 .
\end{aligned}
$$

The observed values of $D_{i}^{\delta}(t, T)$ for $i=1,2,3$ and for our time window are the following:

Term structure of defaultable bonds for the time window $[0,3]$.

GRADE 1: $D_{1}^{\delta}(0,0)=1$.

$$
\begin{aligned}
& D_{1}^{\delta}(0,1)=0.894, D_{1}^{\delta}(1,1)=1 . \\
& D_{1}^{\delta}(0,2)=0.81, \quad D_{1}^{\delta}(1,2)=0.882, D_{1}^{\delta}(2,2)=1 . \\
& D_{1}^{\delta}(0,3)=0.73, \quad D_{1}^{\delta}(1,3)=0.78, D_{1}^{\delta}(2,3)=0.845, D_{1}^{\delta}(3,3)=1 .
\end{aligned}
$$

GRADE 2: $D_{2}^{\delta}(0,0)=1$.

$$
\begin{aligned}
& D_{2}^{\delta}(0,1)=0.884, D_{2}^{\delta}(1,1)=1 . \\
& D_{2}^{\delta}(0,2)=0.78, \quad D_{2}^{\delta}(1,2)=0.875, D_{2}^{\delta}(2,2)=1 . \\
& D_{2}^{\delta}(0,3)=0.70, \quad D_{2}^{\delta}(1,3)=0.75, D_{2}^{\delta}(2,3)=0.82, D_{2}^{\delta}(3,3)=1 .
\end{aligned}
$$

GRADE 3: $D_{3}^{\delta}(0,0)=1$.

$$
\begin{aligned}
& D_{3}^{\delta}(0,1)=0.875, D_{3}^{\delta}(1,1)=1 . \\
& D_{3}^{\delta}(0,2)=0.72, \quad D_{3}^{\delta}(1,2)=0.85, D_{3}^{\delta}(2,2)=1 . \\
& D_{3}^{\delta}(0,3)=0.65, \quad D_{3}^{\delta}(1,3)=0.71, D_{3}^{\delta}(2,3)=0.78, D_{3}^{\delta}(3,3)=1
\end{aligned}
$$

In the above, the physical architecture of the defaultable bonds in the arbitrage free Market could be observed.

The value of $\delta$ is 0.3 . The estimated transition probabilities for the inhomogeneous semi-Markov model available from historical data are the following:

(a) The transition probabilities sequence of the real world is $\{\mathbf{P}(t)\}_{t=0}^{2}$ and are the following:

$$
\begin{gathered}
\mathbf{P}(0)=\left(\begin{array}{cccc}
0.7 & 0.15 & 0.05 & 0.01 \\
0.10 & 0.65 & 0.1 & 0.15 \\
0.08 & 0.12 & 0.55 & 0.25 \\
0 & 0 & 0 & 1
\end{array}\right), \mathbf{P}(1)=\left(\begin{array}{cccc}
0.72 & 0.12 & 0.06 & 0.1 \\
0.08 & 0.67 & 0.11 & 0.14 \\
0.04 & 0.10 & 0.53 & 0.33 \\
0 & 0 & 0 & 1
\end{array}\right), \\
\mathbf{P}(2)=\left(\begin{array}{cccc}
0.66 & 0.14 & 0.07 & 0.13 \\
0.09 & 0.60 & 0.12 & 0.19 \\
0.05 & 0.11 & 0.50 & 0.34 \\
0 & 0 & 0 & 1
\end{array}\right) .
\end{gathered}
$$

(b) The sequence of the real world duration probabilities is 
For $t=0$

$$
\begin{gathered}
\mathbf{H}(0,1)=\left(\begin{array}{cccc}
0.25 & 0.2 & 0.23 & 0.15 \\
0.18 & 0.21 & 0.24 & 0.17 \\
0.16 & 0.14 & 0.25 & 0.21 \\
0 & 0 & 0 & 1
\end{array}\right), \mathbf{H}(0,2)=\left(\begin{array}{cccc}
0.18 & 0.17 & 0.2 & 0.30 \\
0.15 & 0.18 & 0.21 & 0.33 \\
0.14 & 0.12 & 0.18 & 0.36 \\
0 & 0 & 0 & 0
\end{array}\right), \\
\mathbf{H}(0,3)=\left(\begin{array}{cccc}
0.128 & 0.144 & 0.125 & 0.35 \\
0.147 & 0.096 & 0.144 & 0.38 \\
0.128 & 0.063 & 0.125 & 0.041 \\
0 & 0 & 0 & 0
\end{array}\right) .
\end{gathered}
$$

For $t=1$

$$
\mathbf{H}(1,1)=\left(\begin{array}{cccc}
0.24 & 0.18 & 0.22 & 0.16 \\
0.19 & 0.20 & 0.21 & 0.24 \\
0.18 & 0.16 & 0.23 & 0.28 \\
0 & 0 & 0 & 1
\end{array}\right), \mathbf{H}(1,2)=\left(\begin{array}{cccc}
0.19 & 0.21 & 0.24 & 0.22 \\
0.18 & 0.22 & 0.17 & 0.23 \\
0.18 & 0.21 & 0.20 & 0.19 \\
0 & 0 & 0 & 0
\end{array}\right),
$$

For $t=2$

$$
\mathbf{H}(2,1)=\left(\begin{array}{cccc}
0.2 & 0.12 & 0.21 & 0.23 \\
0.17 & 0.18 & 0.20 & 0.27 \\
0.16 & 0.15 & 0.20 & 0.35 \\
0 & 0 & 0 & 1
\end{array}\right)
$$

We are interested for our purposes in finding the sequence of forward probabilities ${ }_{F} \mathbf{E}(t, t+m)$ for $t=0,1,2$ and $m=1,2,3$. Applying the data above in the Algorithm 8.1, we provide the values of the forward probabilities together with the relative real word entrance probabilities:

$$
\begin{aligned}
& { }_{F} \mathbf{E}(0,1)=\left(\begin{array}{cccc}
0.12 & 0.02 & 0.01 & 0.01 \\
0.02 & 0.13 & 0.02 & 0.03 \\
0.01 & 0.01 & 0.10 & 0.40 \\
0 & 0 & 0 & 1
\end{array}\right), \mathbf{E}(0,1)=\left(\begin{array}{cccc}
0.18 & 0.03 & 0.01 & 0.02 \\
0.02 & 0.14 & 0.03 & 0.03 \\
0.02 & 0.02 & 0.14 & 0.06 \\
0 & 0 & 0 & 1
\end{array}\right) \\
& { }_{F} \mathbf{E}(1,2)=\left(\begin{array}{cccc}
0.08 & 0.01 & 0.01 & 0.01 \\
0.01 & 0.09 & 0.02 & 0.02 \\
0.01 & 0.02 & 0.08 & 0.06 \\
0 & 0 & 0 & 1
\end{array}\right), \mathbf{E}(1,2)=\left(\begin{array}{cccc}
0.15 & 0.03 & 0.02 & 0.04 \\
0.02 & 0.14 & 0.02 & 0.06 \\
0.01 & 0.02 & 0.11 & 0.11 \\
0 & 0 & 0 & 1
\end{array}\right) \\
& { }_{F} \mathbf{E}(2,3)=\left(\begin{array}{cccc}
0.11 & 0.02 & 0.01 & 0.03 \\
0.02 & 0.14 & 0.03 & 0.07 \\
0.01 & 0.02 & 0.12 & 0.13 \\
0 & 0 & 0 & 1
\end{array}\right), \mathbf{E}(2,3)=\left(\begin{array}{cccc}
0.13 & 0.02 & 0.02 & 0.03 \\
0.02 & 0.11 & 0.02 & 0.05 \\
0.01 & 0.02 & 0.11 & 0.11 \\
0 & 0 & 0 & 1
\end{array}\right) \\
& { }_{F} \mathbf{E}(0,2)=\left(\begin{array}{cccc}
0.37 & 0.08 & 0.03 & 0.09 \\
0.04 & 0.33 & 0.06 & 0.13 \\
0.01 & 0.02 & 0.13 & 0.11 \\
0 & 0 & 0 & 1
\end{array}\right), \mathbf{E}(0,2)=\left(\begin{array}{cccc}
0.15 & 0.03 & 0.01 & 0.04 \\
0.02 & 0.14 & 0.03 & 0.06 \\
0.01 & 0.02 & 0.11 & 0.10 \\
0 & 0 & 0 & 1
\end{array}\right) \\
& { }_{F} \mathbf{E}(1,3)=\left(\begin{array}{cccc}
0.14 & 0.04 & 0.02 & 0.03 \\
0.06 & 0.35 & 0.04 & 0.09 \\
0.01 & 0.03 & 0.13 & 0.09 \\
0 & 0 & 0 & 1
\end{array}\right), \mathbf{E}(1,3)=\left(\begin{array}{cccc}
0.24 & 0.05 & 0.04 & 0.03 \\
0.06 & 0.22 & 0.05 & 0.04 \\
0.02 & 0.04 & 0.17 & 0.08 \\
0 & 0 & 0 & 1
\end{array}\right) \\
& { }_{F} \mathbf{E}(0,3)=\left(\begin{array}{cccc}
0.13 & 0.05 & 0.01 & 0.05 \\
0.02 & 0.11 & 0.03 & 0.06 \\
0.01 & 0.02 & 0.06 & 0.08 \\
0 & 0 & 0 & 1
\end{array}\right), \mathbf{E}(0,3)=\left(\begin{array}{cccc}
0.15 & 0.04 & 0.02 & 0.05 \\
0.04 & 0.11 & 0.03 & 0.07 \\
0.02 & 0.02 & 0.11 & 0.12 \\
0 & 0 & 0 & 1
\end{array}\right)
\end{aligned}
$$


Funding: This research received no external funding.

Institutional Review Board Statement: Not applicable.

Informed Consent Statement: Not applicable.

Data Availability Statement: No real data were used, only representative data.

Conflicts of Interest: The author declares no conflict of interest.

\section{References}

1. Carty, L.V.; Fons, J.S. Measuring changes in corporate credit quality. J. Fixed Income 1994, 4, 27-41. [CrossRef]

2. Duffie, D.; Saita, L.; Wang, K. Multiperiod corporate default prediction with stochastic covariates. J. Financ. Econ. 2007, 83, 635-665. [CrossRef]

3. Vasileiou, A.; Vassiliou, P.-C.G. An inhomogeneous semi-Markov model for the term Stucture of credit risk spreads. Adv. Appl. Prob. 2006, 38, 171-198. [CrossRef]

4. D'Amico, G.; Janssen, J.; Manca, R. Homogeneous semi-Markov reliability models for credit risk management. Des. Econ. Finan. 2005, 28, 79-93. [CrossRef]

5. Howard, D.T. Dynamic Probabilistic Systems. Vol. I and II; John Wiley: Hoboken, NJ, USA, 1971.

6. D'Amico, G.; Janssen, J.; Manca, R. Valuing credit default swap in a non-homogeneous semi-Markov rating based model. Comput. Econom. 2007, 29, 119-138. [CrossRef]

7. D'Amico, G.; Janssen, J.; Manca, R. Discete time non-homogeneous semi Markov reliability transition credit risk models and the default distributions functions. Comput. Econom. 2011, 38, 465-481. [CrossRef]

8. D'Amico, G. A semi-Markov maintainance model with credit rating application. IMA J. Managem. Math. 2009, 20, 51-58. [CrossRef]

9. D'Amico, G.; Janssen, J.; Manca, R. Initial and final backward and forward discrete time non-homogeneous semi-Markov credit risk models. Methodol. Comput. Appl. Prob. 2010, 12, 215-225. [CrossRef]

10. D'Amico, G.; Janssen, J.; Manca, R. Downward migration credit risk problem: A non-homogeneous backward semi-Markov reliability approach. J. Oper. Res. Soc. 2016, 67, 393-401. [CrossRef]

11. Vassiliou, P.-C.G.; Vasileiou, A. Asymptotic behaviour of the survival probabilities in an inhomogeneous semi-Markov model for the migration process in credit risk. Linear Algebra Its Appl. 2013, 438, 2880-2903. [CrossRef]

12. Vassiliou, P.-C.G. Fuzzy semi-Markov migration process in credit risk. Fuzzy Sets Syst. 2013, 223, 39-58. [CrossRef]

13. Huang, J. Review on term structure. In Proceedings of the 6th International Conference on Electronic, Mechanical, Information and Management Society, Shenyang, China, 1-3 April 2016; Volume 40, pp. 1278-1281.

14. D'Amico, G.; Manca, R.; Salvi, G. Bivariate semi-Markov reward chain and credit spreads. IMA J. Manag. Math. 2016, 27, 529-556. [CrossRef]

15. D'Amico, G.; Lika, A.; Petroni, F. Risk management of pention fund: A model for salary evolution. Int. J. Financ. Stud. 2019, 7, 44. [CrossRef]

16. D'Amico, G. Stochastic dividend discount model: Risc and return. Markov Process. Relat. Fields 2017, $23,349-376$.

17. Magni, C.; Magnoli, S.; Marchioni, A. Rating firms and sensitivity analysis. J. Oper. Res. 2019, 71, 1940-1958. [CrossRef]

18. Wu, L.; Zhuang, Y.; Li, W. A new default intensity model with Fuzziness and hesitation. Int. J. Comput. Intell. Syst. 2016, 9, 340-350. [CrossRef]

19. Wu, L.; Mei, X.; Sun, J. A new default probability calculation formula and its application under uncertain enviroments. Discret. Dyn. Nat. Soc. 2018, 2018, 3481863. [CrossRef]

20. Puneet, P.; Selvamuthu, D.; D'Amico, G.; Manca, R. Portfolio optimization of credit risky bonds: A semi-Markov approach. Financ. Innov. 2020, 6, 25.

21. De Blassis, R. The price leadership share: A new measure of price discovery in financial markets. Ann. Financ. 2020, 16, 381-405. [CrossRef]

22. D'Amico, G.; Manca, R.; Salvi, G. Bivariate semi-Markov process for counterparty credit risk. Commun. Stat. Theory Methods 2014, 43, 1503-1522. [CrossRef]

23. Vassiliou, P.-C.G. Semi-Markov migration process in a stochastic market in credit risk. Linear Algebra Its Appl.2014, 450, 13-43. [CrossRef]

24. Lando, D.; Skodeberg, T.M. Analyzing rating transitions and rating drift with continuous observations. J. Bank. Financ. 2002, 26, 423-444. [CrossRef]

25. Bingham, N.H.; Kiesel, R. Risk-Neutral Valuation, Pricing and Hedging Financial Derivatives; Springer: London, UK, 1998.

26. Shreve, S.E. Stochastic Calculus for Finance, Vol II, Continuous-Time Models; Springer: New York, NY, USA, 2004.

27. Vassiliou, P.-C.G. Discrete-Time Asset Pricing Models in Applied Stochastic Finance; John Wiley: Hoboken, NJ, USA, 2010

28. Bielecki, T.R.; Rutkowski, M. Credit Risk: Modeling, Valuation, and Hedging; Springer: Berlin, Germany, 2002.

29. Vassiliou, P.-C.G.; Papadopoulou, A.A. Nonhomogeneous semi-Markov systems and maintainability of the state sizes. J. Appl. Prob. 1992, 29, 519-534. [CrossRef]

30. Pyke, R. Markov Renewal Processes with Finitely Many States. Ann. Math. Stat.1961, 32, 1243-1259. [CrossRef] 
31. Musiela, M.; Rutkowski, M. Martingale Methods in Financial Modelling; Springer Science: Berlin/Heidelberg, Germany, 1997.

32. Lee, E.T. Statistical Methods for Survival Data Analysis, 2nd ed.; John Wiley: Hoboken, NJ, USA, 2003.

33. McClean, S.I.; Gribbin, J.O. Estimation for incomplete manpower data. Appl. Stoch. Models Data Anal. 1987, 3, 13-25. [CrossRef]

34. McClean, S.I.; Gribbin, J.O. A non-parametric competing risks model for manpower planning. Appl. Stoch. Models Data Anal. 1991, 7, 327-341. [CrossRef]

35. McClean, S.I.; Montgomery, E.; Ugwuowo, F. Inhomogeneous continuous-time Markov and semi-Markov manpower models. Appl. Stoch. Models Data Anal. 1997, 13, 191-198. [CrossRef]

36. McClean, S.I.; Montgomery, E. Estimation for semi-Markov manpower models in a stochastic enviroment. In Semi-Markov Models and Applications; Janssen, J., Limnios, N., Eds.; Kluwer: Alphen aan den Rijn, The Netherland, 1999; pp. $219-227$.

37. Crouchy, M.; Galai, D.; Mark, R. Prototype risk rating system. J. Bank. Financ. 2001, 25, 27-41. 